This is an Open Access article, distributed under the terms of the Creative Commons Attribution licence (http://creativecommons.org/licenses/by/4.0/), which permits unrestricted re-use, distribution, and reproduction in any medium, provided the original work is properly cited.

doi:10.1017/jfm.2016.821

\title{
Viscous propulsion in active transversely isotropic media
}

\author{
G. Cupples ${ }^{1}$, R. J. Dyson ${ }^{1}$ and D. J. Smith ${ }^{1,2,3, \dagger}$ \\ ${ }^{1}$ School of Mathematics, University of Birmingham, Birmingham B15 2TT, UK \\ ${ }^{2}$ Institute for Metabolism and Systems Research, University of Birmingham, Birmingham B15 2TT, UK \\ ${ }^{3}$ Centre for Human Reproductive Science, Birmingham Women's NHS Foundation Trust, \\ Birmingham B15 2TG, UK
}

(Received 3 August 2016; revised 21 November 2016; accepted 30 November 2016; first published online 5 January 2017)

Taylor's swimming sheet is a classical model of microscale propulsion and pumping. Many biological fluids and substances are fibrous, having a preferred direction in their microstructure; for example, cervical mucus is formed of polymer molecules which create an oriented fibrous network. Moreover, suspensions of elongated motile cells produce a form of active oriented matter. To understand how these effects modify viscous propulsion, we extend Taylor's classical model of small-amplitude zero-Reynolds-number propulsion of a 'swimming sheet' via the transversely isotropic fluid model of Ericksen, which is linear in strain rate and possesses a distinguished direction. The energetic costs of swimming are significantly altered by all rheological parameters and the initial fibre angle. Propulsion in a passive transversely isotropic fluid produces an enhanced mean rate of working, independent of the initial fibre orientation, with an approximately linear dependence of the energetic cost on the extensional and shear enhancements to the viscosity caused by fibres. In this regime, the mean swimming velocity is unchanged from the Newtonian case. The effect of the constant term in Ericksen's model for the stress, which can be identified as a fibre tension or alternatively a stresslet characterising an active fluid, is also considered. This stress introduces an angular dependence and dramatically changes the streamlines and flow field; fibres aligned with the swimming direction increase the energetic demands of the sheet. The constant fibre stress may result in a reversal of the mean swimming velocity and a negative mean rate of working if it is sufficiently large relative to the other rheological parameters.

Key words: micro-organism dynamics, propulsion, swimming/flying

\section{Introduction}

Large organisms propel themselves through a fluid by utilising the inertia of the surrounding fluid. For very small organisms and cells swimming at low Reynolds 


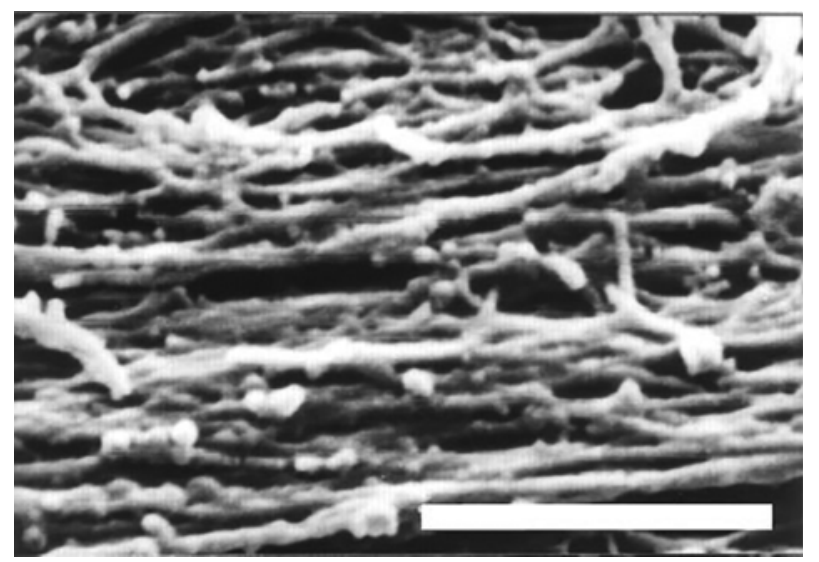

FIgURE 1. Parallel filament mesh in cervical mucus during the time of ovulation; bar = $10 \mu \mathrm{m}$. Republished with permission of Oxford University Press, from 'Ultrastructure of the Human Periovulatory Cervical Mucus', Ceric et al. (2005); permission conveyed through Copyright Clearance Center, Inc.

numbers, inertial propulsion is not possible (Fauci \& Dillon 2006; Lauga \& Powers 2009); time-reversible kinematics result in no net displacement for the small body. Taylor's 'swimming sheet' is one of the classical models of zero-Reynolds-number swimming; time-reversal symmetry is broken by the wave direction.

Many of the biological fluids in which these cells and organisms swim are non-Newtonian; hence, modelling of swimming in such fluids is of interest. The present study is motivated by the fibrous nature of many biological media, for example, the cervical mucus encountered by the spermatozoa of many internally fertilising species, and active suspensions of elongated cells. Throughout the menstrual cycle, the rheology of cervical mucus changes due to hormonally induced variations in hydration and associated changes in the glycofilament mucin structure. During ovulation, these fibres form a parallel network (figure 1), and sperm migration occurs through this glycofilament structure (Chrétien 1982; Ceric, Silva \& Vigil 2005). It is therefore of great interest to determine how Stokesian swimming is modified by the presence of an aligned fibrous network.

Taylor's pioneering study of Stokesian swimming consists of an infinite sheet undergoing waves of lateral displacement (figure 2). This model was formulated as the far-field Stokes flow produced by a swimming motion given by a small-amplitude sinusoidal wave, and the associated mean rate of working was calculated as a measure of the energetic cost of swimming. Subsequent studies included a 3D model of a waving cylindrical tail (Taylor 1952), investigations by other authors into larger-amplitude motion (Drummond 1966) and more recently the unsteady Stokes flow problem (Pak \& Lauga 2010).

Generalisation of Taylor's model to non-Newtonian fluids has been an area of significant interest; for a detailed review see Lauga \& Powers (2009). Chaudhury (1979) initially extended the model to incorporate viscoelastic fluids; it was found that the properties of the fluid lead to an increased steady swimming velocity for lower Reynolds numbers. This problem was reconsidered more recently by Lauga (2007), who deduced that the mean swimming velocity in a nonlinear viscoelastic fluid is reduced relative to that in a Newtonian fluid; in certain cases the swimming 


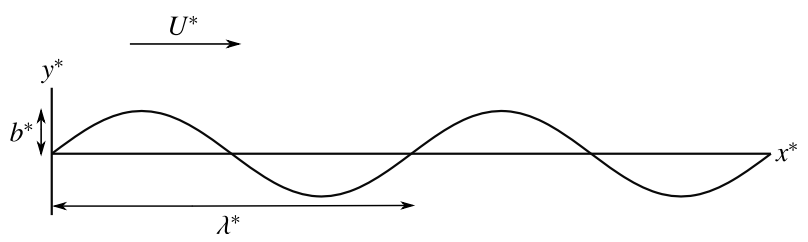

FIGURE 2. A schematic of Taylor's swimming sheet in a Newtonian fluid. Working in a frame of reference in which the sheet is stationary, $b^{*}$ is the amplitude, $\lambda^{*}=2 \pi / k^{*}$ is the wavelength and $k^{*}$ is the wavenumber. The flow at infinity in the $x^{*}$-direction is $U^{*}$. A travelling wave traverses the sheet with speed $c^{*}=\omega^{*} / k^{*}$, where $\omega^{*}$ is the angular velocity.

direction is reversed (Fu, Powers \& Wolgemuth 2007); see also Fu, Wolgemuth \& Powers (2009) and Teran, Fauci \& Shelley (2010). Vélez-Cordero \& Lauga (2013) found propulsion in shear-thinning fluids to be more efficient than that in Newtonian or shear-thickening fluids. Riley \& Lauga (2014) modelled active propulsion with fluid-structure interaction, and in a subsequent study deduced that for multiple travelling waves, the mean swimming velocity of the sheet is enhanced (Riley \& Lauga 2015). Further to this, Krieger, Spagnolie \& Powers (2014), Krieger, Dias \& Powers (2015a) and Krieger, Spagnolie \& Powers (2015b) considered how liquid crystals affect the swimming of micro-organisms. Steady-state and startup models for hexatic liquid crystals were considered along with a nematic steady-state model.

A transversely isotropic fluid exhibits a (perhaps spatially and temporally varying) preferred direction, and has been used to model fibre-reinforced fluids. Previous applications include the mechanical behaviour of collagen gel, the growth of plant root cell walls, suspensions of biomolecules and a multiphase model of extracellular matrix (Green \& Friedman 2008; Dyson \& Jensen 2010; Dyson et al. 2015; Holloway, Dyson \& Smith 2015). These models comprise a modified constitutive equation describing a viscous fluid with suspended aligned fibres and an expression for the evolution of fibre orientation. A transversely isotropic fluid also provides a model of 'active' suspensions of elongated swimmers (Holloway et al. 2016).

In this study, we consider swimming in transversely isotropic fluids via the constitutive law of Ericksen (1960). In $\S 2$, we introduce the governing equations associated with transversely isotropic fluids. The problem is solved in $\S 3$, solving for the leading-order velocity field, mean swimming velocity at next order and mean rate of working. The dependence of these quantities on the rheological parameters is explored in $\S 4$ and discussed in $\S 5$.

\section{Governing equations}

The incompressibility and generalised Navier-Stokes equations are

$$
\begin{gathered}
\nabla^{*} \cdot \boldsymbol{u}^{*}=0 \\
\rho^{*}\left(\frac{\partial \boldsymbol{u}^{*}}{\partial t^{*}}+\left(\boldsymbol{u}^{*} \cdot \nabla^{*}\right) \boldsymbol{u}^{*}\right)=\nabla^{*} \cdot \sigma^{*},
\end{gathered}
$$

where $\boldsymbol{u}^{*}=\left(u^{*}, v^{*}\right)$ is the velocity, $\rho^{*}$ is the density and $\boldsymbol{\sigma}^{*}$ is the stress tensor. We work in a $2 \mathrm{D}$ Cartesian coordinate system $\left(x^{*}, y^{*}\right)$; asterisk notation represents dimensional variables. A constitutive law is also required for $\boldsymbol{\sigma}^{*}$, which we prescribe in the next section. 


\subsection{Transversely isotropic stress tensor}

Ericksen's (1960) model consists of a stress tensor which is linear in strain rate and depends on a unit vector $\boldsymbol{a}$ describing the fibre orientation; this model takes the simplest form that satisfies the required invariances,

$$
\sigma_{i j}^{*}=-p^{*} \delta_{i j}+2 \mu^{*} e_{i j}^{*}+\mu_{1}^{*} a_{i} a_{j}+\mu_{2}^{*} a_{i} a_{j} a_{k} a_{l} e_{k l}^{*}+2 \mu_{3}^{*}\left(a_{l} a_{i} e_{l j}^{*}+a_{m} a_{j} e_{i m}^{*}\right) .
$$

We define $p^{*}$ as the pressure, $\delta_{i j}$ as the Kronecker delta function and $e_{i j}^{*}=\left(\partial u_{i}^{*} / \partial x_{j}^{*}+\right.$ $\left.\partial u_{j}^{*} / \partial x_{i}^{*}\right) / 2$ as the rate-of-strain tensor (Ericksen 1960).

By considering simple flows with a uniform director field, we may interpret the rheological parameters as follows. By setting $\mu_{1}^{*}=\mu_{2}^{*}=\mu_{3}^{*}=0$, the stress tensor for an incompressible Newtonian fluid remains, with 'matrix viscosity' $\mu^{*}$ (Holloway et al. 2015). The term with $\mu_{1}^{*}$ has no dependence on velocity, suggesting that $\mu_{1}^{*}$ relates to a tension in the fibre direction (Dyson \& Jensen 2010). This term can also be related to the stresslet-type active behaviour of fibres in a perfectly aligned active fluid (Holloway et al. 2016). We will therefore refer to this quantity as the active parameter. This term can be taken as a simple model for suspensions of self-propelling microscopic bodies such as bacteria or active gels of molecular motor proteins. The viscosity associated with extensional flow parallel to the fibre direction is $\mu_{\|}^{*}=\mu^{*}+\left(\mu_{2}^{*}+4 \mu_{3}^{*}\right) / 2$, the viscosity associated with the flow orthogonal to the fibre direction is $\mu_{\perp}^{*}=\mu^{*}$ and the viscosity of shear flow in the fibre direction is $\mu_{s}^{*}=\mu^{*}+\mu_{3}^{*}$ (Dyson \& Jensen 2010). Since $\mu_{2}^{*}$ only has an impact on extensional viscosity parallel to the fibre direction, $\mu_{\|}^{*}$, it is termed the anisotropic extensional viscosity. The parameter $\mu_{3}^{*}$ distinguishes $\mu_{\perp}^{*}$ from $\mu_{s}^{*}$ and so is labelled the anisotropic shear viscosity; this parameter represents the difference between shear viscosities parallel and perpendicular to the fibre direction (Green \& Friedman 2008; Dyson \& Jensen 2010; Holloway et al. 2015).

\subsection{Fibre evolution equation}

A fibre evolution equation describes the evolution of fibre orientation with time. We use the form given by Green \& Friedman (2008),

$$
\frac{\partial \boldsymbol{a}}{\partial t^{*}}+\boldsymbol{u}^{*} \cdot \nabla^{*} \boldsymbol{a}+\left[\boldsymbol{a} \cdot\left(\boldsymbol{a} \cdot \nabla^{*} \boldsymbol{u}^{*}\right)\right] \boldsymbol{a}=\boldsymbol{a} \cdot \nabla^{*} \boldsymbol{u}^{*},
$$

which corresponds to a specific case of Ericksen's (1960) equation in the long-fibre limit. It should be noted that $|\boldsymbol{a}|=1$, and thus the model only considers local alignment of fibres and not their length. This gives a generalised form of Jeffery's treatment for long ellipsoidal particles aligning with flow (Jeffery 1922; Dyson \& Jensen 2010).

Since $\boldsymbol{a} \cdot \boldsymbol{a}=1$, the component of (2.4) in the $\boldsymbol{a}$-direction is automatically satisfied. The orthogonal component of (2.4) is

$$
\boldsymbol{a}^{\perp} \cdot\left[\frac{\partial \boldsymbol{a}}{\partial t^{*}}+\boldsymbol{u}^{*} \cdot \nabla^{*} \boldsymbol{a}+\left[\boldsymbol{a} \cdot\left(\boldsymbol{a} \cdot \nabla^{*} \boldsymbol{u}^{*}\right)\right] \boldsymbol{a}-\boldsymbol{a} \cdot \nabla^{*} \boldsymbol{u}^{*}\right]=0,
$$

where $\boldsymbol{a}^{\perp}$ is a unit vector perpendicular to $\boldsymbol{a}$.

\subsection{Boundary conditions}

We work in a frame of reference moving with the swimmer in the $x^{*}$-direction; the horizontal flow as $y^{*} \rightarrow \infty$ therefore gives the mean swimming velocity. No-slip 
conditions on the sheet, representing a travelling wave with speed $c^{*}=\omega^{*} / k^{*}$, are thus

$$
u^{*}=0, \quad v^{*}=-\omega^{*} b^{*} \cos \left(k^{*} x^{*}-\omega^{*} t^{*}\right), \quad \text { on } y^{*}=y_{s}^{*}=b^{*} \sin \left(k^{*} x^{*}-\omega^{*} t^{*}\right) .
$$

The parameter $b^{*}$ is the amplitude, $k^{*}$ is the wavenumber, $y_{s}^{*}$ is the equation of the sheet surface and $\lambda^{*}=2 \pi / k^{*}$ is the wavelength. The velocity must remain bounded as $y^{*} \rightarrow \infty$.

\subsection{Non-dimensionalisation}

The model is non-dimensionalised as follows:

$$
\boldsymbol{u}^{*}=\frac{\omega^{*}}{k^{*}} \boldsymbol{u}, \quad \boldsymbol{x}^{*}=\frac{\boldsymbol{x}}{k^{*}}, \quad t^{*}=\frac{t}{\omega^{*}}, \quad p^{*}=\omega^{*} \mu^{*} p, \quad \boldsymbol{\sigma}^{*}=\omega^{*} \mu^{*} \boldsymbol{\sigma} .
$$

The continuity and fibre evolution equations are unchanged. For microscopic swimmers, the Reynolds number, $R e=\rho^{*} \omega^{*} / k^{* 2} \mu^{*}$, is much less than 1 , so we neglect inertial terms. The resulting system of partial differential equations is therefore

$$
\begin{gathered}
\nabla \cdot \boldsymbol{u}=0, \\
\nabla \cdot \sigma=\mathbf{0}, \\
\boldsymbol{a}^{\perp} \cdot\left[\frac{\partial \boldsymbol{a}}{\partial t}+\boldsymbol{u} \cdot \nabla \boldsymbol{a}+[\boldsymbol{a} \cdot(\boldsymbol{a} \cdot \nabla \boldsymbol{u})] \boldsymbol{a}-\boldsymbol{a} \cdot \nabla \boldsymbol{u}\right]=0,
\end{gathered}
$$

where

$$
\sigma_{i j}=-p \delta_{i j}+2 e_{i j}+\mu_{1} a_{i} a_{j}+\mu_{2} a_{i} a_{j} a_{k} a_{l} e_{k l}+2 \mu_{3}\left(a_{l} a_{i} e_{l j}+a_{m} a_{j} e_{i m}\right),
$$

with dimensionless groups

$$
\mu_{1}=\frac{\mu_{1}^{*}}{\mu^{*} \omega^{*}}, \quad \mu_{2}=\frac{\mu_{2}^{*}}{\mu^{*}}, \quad \mu_{3}=\frac{\mu_{3}^{*}}{\mu^{*}} .
$$

The boundary conditions (2.6) become

$$
u=0, \quad v=-\varepsilon \cos (x-t), \quad \text { on } y=y_{s}=\varepsilon \sin (x-t),
$$

where $\varepsilon=k^{*} b^{*} \ll 1$. Moreover, $u$ and $v$ must remain bounded as $y \rightarrow \infty$.

Four regimes in parameter space, depicted in figure 3 , will be considered in our results: (i) a passive transversely isotropic fluid, occurring when $\mu_{1}=0$; (ii) an active fluid ( $\mu_{1}$ non-zero) with $\mu_{2}=\mu_{3}=0$; (iii) a nearly isotropic regime, where all parameters take values up to 5; (iv) the regime where at least one of $\mu_{1}, \mu_{2}$ and $\mu_{3}$ is much larger than 1 . It should be noted that $\mu_{1}$ may be positive or negative, representing active 'puller' or 'pusher' behaviour respectively (Saintillan 2010).

\section{Asymptotic solution}

\subsection{Stream function formulation}

To determine the effect of fibres on the mean swimming velocity, we consider an initially spatially uniform fibre angle, $\phi$, aligned such that $\boldsymbol{a}(x, y, 0)=(\cos \phi, \sin \phi)$. 


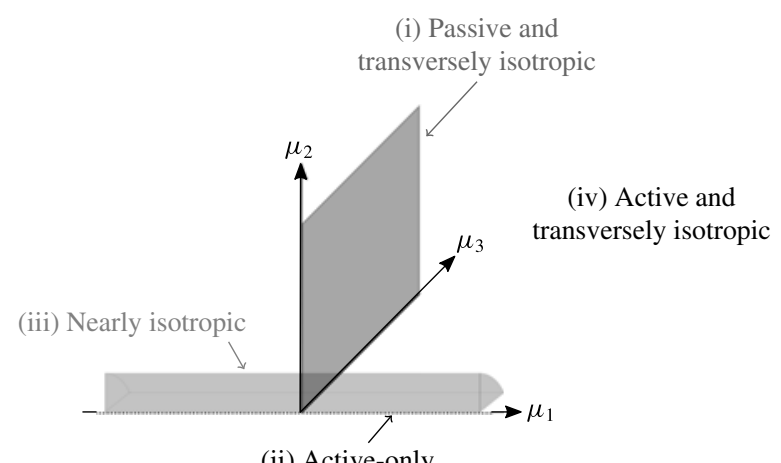

(ii) Active-only

FIGURE 3. Regimes of interest in the parameter space: (i) the plane $\mu_{1}=0$ is the passive transversely isotropic regime; (ii) the dashed line represents the active-only regime where $\mu_{2}=\mu_{3}=0$; (iii) the quarter cylinder is the nearly isotropic regime where neither $\mu_{2}$ nor $\mu_{3}$ is large; (iv) the remaining region is the fully active and transversely isotropic regime.

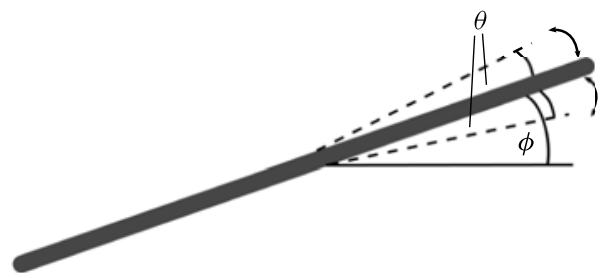

FIGURE 4. A schematic showing the initial uniform orientation angle, $\phi$, and the small perturbation away from this angle, $\theta$.

As the sheet swims, this alignment will evolve, initially as a small perturbation $\theta(x, y, t)$ such that

$$
\begin{aligned}
\boldsymbol{a} & =(\cos (\phi+\theta), \sin (\phi+\theta)) \\
& \approx(\cos \phi-\theta \sin \phi, \sin \phi+\theta \cos \phi)
\end{aligned}
$$

(see figure 4). The components of the stress tensor in terms of $\phi$ and $\theta$ are given in appendix A. Taking the curl of (2.9) eliminates pressure, reducing (2.9) to a single equation. Since the flow is incompressible, we introduce a dimensionless stream function

$$
u=\frac{\partial \psi}{\partial y}, \quad v=-\frac{\partial \psi}{\partial x},
$$

transforming (2.9) to

$$
\begin{aligned}
(1 & \left.+\frac{\mu_{2}}{4} \sin ^{2} 2 \phi+\mu_{3}\right) \nabla^{4} \psi-\mu_{1}\left[2 \operatorname { s i n } 2 \phi \left(\theta\left(\frac{\partial^{2} \theta}{\partial y^{2}}-\frac{\partial^{2} \theta}{\partial x^{2}}\right)+\left(\frac{\partial \theta}{\partial y}\right)^{2}-\left(\frac{\partial \theta}{\partial x}\right)^{2}\right.\right. \\
& \left.\left.+\frac{\partial^{2} \theta}{\partial x \partial y}\right)+\cos 2 \phi\left(\frac{\partial^{2} \theta}{\partial x^{2}}-\frac{\partial^{2} \theta}{\partial y^{2}}+4\left(\frac{\partial \theta}{\partial x} \frac{\partial \theta}{\partial y}+\theta \frac{\partial^{2} \theta}{\partial x \partial y}\right)\right)\right] \\
& -\mu_{2}\left[\operatorname { s i n } 4 \phi \left(-\frac{\theta}{2}\left(\frac{\partial^{4} \psi}{\partial x^{4}}-3 \frac{\partial^{4} \psi}{\partial x^{2} \partial y^{2}}+\frac{\partial^{4} \psi}{\partial y^{4}}\right)+\frac{1}{2}\left(\frac{\partial^{2} \theta}{\partial x^{2}}-\frac{\partial^{2} \theta}{\partial y^{2}}\right)\left(\frac{\partial^{2} \psi}{\partial y^{2}}-\frac{\partial^{2} \psi}{\partial x^{2}}\right)\right.\right.
\end{aligned}
$$




$$
\begin{aligned}
& -\frac{\partial \theta}{\partial y}\left(\frac{\partial^{3} \psi}{\partial y^{3}}-3 \frac{\partial^{3} \psi}{\partial x^{2} \partial y}\right)+2 \frac{\partial^{2} \theta}{\partial x \partial y} \frac{\partial^{2} \psi}{\partial x \partial y}+\frac{\partial \theta}{\partial x}\left(3 \frac{\partial^{3} \psi}{\partial x \partial y^{2}}-\frac{\partial^{3} \psi}{\partial x^{3}}\right) \\
& \left.+\theta \frac{\partial^{4} \psi}{\partial x^{2} \partial y^{2}}+\frac{1}{2}\left(\frac{\partial^{4} \psi}{\partial x^{3} \partial y}-\frac{\partial^{4} \psi}{\partial x \partial y^{3}}\right)\right)+\cos 4 \phi\left(2 \theta\left(\frac{\partial^{4} \psi}{\partial x^{3} \partial y}-\frac{\partial^{4} \psi}{\partial x \partial y^{3}}\right)\right. \\
& +\left(\frac{\partial^{2} \theta}{\partial x^{2}}-\frac{\partial^{2} \theta}{\partial y^{2}}\right) \frac{\partial^{2} \psi}{\partial x \partial y}-\frac{\partial \theta}{\partial x}\left(\frac{\partial^{3} \psi}{\partial y^{3}}-3 \frac{\partial^{3} \psi}{\partial x^{2} \partial y}\right) \\
& \left.\left.-\frac{\partial \theta}{\partial y}\left(3 \frac{\partial^{3} \psi}{\partial x \partial y^{2}}-\frac{\partial^{3} \psi}{\partial x^{3}}\right)-\frac{\partial^{2} \theta}{\partial x \partial y}\left(\frac{\partial^{2} \psi}{\partial y^{2}}-\frac{\partial^{2} \psi}{\partial x^{2}}\right)-\frac{\partial^{4} \psi}{\partial x^{2} \partial y^{2}}\right)\right]=0 .
\end{aligned}
$$

When $\mu_{1}=\mu_{2}=\mu_{3}=0$, (3.3) reduces to the familiar biharmonic equation of Newtonian Stokes flow. The evolution equation (2.10) becomes

$$
\begin{aligned}
& \frac{\partial \theta}{\partial t}+\left[\frac{\partial \psi}{\partial y} \frac{\partial \theta}{\partial x}-\frac{\partial \psi}{\partial x} \frac{\partial \theta}{\partial y}\right]+\left(\sin ^{2} \phi+\frac{\theta}{2} \sin 2 \phi\right) \frac{\partial^{2} \psi}{\partial y^{2}} \\
& +(\sin 2 \phi+\theta \cos 2 \phi) \frac{\partial^{2} \psi}{\partial x \partial y}+\left(\cos ^{2} \phi-\frac{\theta}{2} \sin 2 \phi\right) \frac{\partial^{2} \psi}{\partial x^{2}} \\
& +\theta\left(\left[(\cos \phi-\theta \sin \phi)^{2}-(\sin \phi+\theta \cos \phi)^{2}\right] \frac{\partial^{2} \psi}{\partial x \partial y}\right. \\
& \left.+(\cos \phi-\theta \sin \phi)(\sin \phi+\theta \cos \phi)\left(\frac{\partial^{2} \psi}{\partial y^{2}}-\frac{\partial^{2} \psi}{\partial x^{2}}\right)\right)=0 .
\end{aligned}
$$

The boundary conditions (2.13) become

$$
\frac{\partial \psi}{\partial y}=0, \quad \frac{\partial \psi}{\partial x}=\varepsilon \cos (x-t), \quad \text { on } y=\varepsilon \sin (x-t),
$$

with $\psi$ having bounded first derivatives as $y \rightarrow \infty$.

\subsection{Perturbation expansion}

To apply the boundary conditions at $y=0$ rather than on the sheet, we make the smallamplitude expansion

$$
\begin{gathered}
\left.\frac{\partial \psi}{\partial y}\right|_{y=0}+\left.\varepsilon \sin (x-t) \frac{\partial^{2} \psi}{\partial y^{2}}\right|_{y=0}+\cdots=0, \\
\left.\frac{\partial \psi}{\partial x}\right|_{y=0}+\left.\varepsilon \sin (x-t) \frac{\partial^{2} \psi}{\partial y \partial x}\right|_{y=0}+\cdots=\varepsilon \cos (x-t) .
\end{gathered}
$$

The velocity and fibre angle perturbations thus take the form

$$
\begin{gathered}
\psi(x, y, t ; \varepsilon)=\varepsilon \psi_{0}(x, y, t)+\varepsilon^{2} \psi_{1}(x, y, t)+\cdots, \\
\theta(x, y, t ; \varepsilon)=\varepsilon \theta_{0}(x, y, t)+\varepsilon^{2} \theta_{1}(x, y, t)+\cdots
\end{gathered}
$$

As in Taylor's analysis, the background flow (i.e. the sheet swimming velocity) will occur at order $\varepsilon^{2}$. 


\subsection{Leading-order solution}

Substituting the expansions into (3.3) and (3.4) and equating coefficients of powers of $\varepsilon$ yields the leading-order partial differential equation. At order $\varepsilon$, (3.3) yields

$$
\begin{aligned}
& \left(1+\frac{\mu_{2}}{4} \sin ^{2} 2 \phi+\mu_{3}\right) \nabla^{4} \psi_{0}-\mu_{1}\left(2 \sin 2 \phi \frac{\partial^{2} \theta_{0}}{\partial x \partial y}+\cos 2 \phi\left(\frac{\partial^{2} \theta_{0}}{\partial x^{2}}-\frac{\partial^{2} \theta_{0}}{\partial y^{2}}\right)\right) \\
& +\mu_{2}\left(\cos 4 \phi \frac{\partial^{4} \psi_{0}}{\partial x^{2} \partial y^{2}}+\frac{\sin 4 \phi}{2}\left(\frac{\partial^{4} \psi_{0}}{\partial x \partial y^{3}}-\frac{\partial^{4} \psi_{0}}{\partial x^{3} \partial y}\right)\right)=0
\end{aligned}
$$

and (3.4) yields

$$
\frac{\partial \theta_{0}}{\partial t}+\sin 2 \phi \frac{\partial^{2} \psi_{0}}{\partial x \partial y}+\cos ^{2} \phi \frac{\partial^{2} \psi_{0}}{\partial x^{2}}+\sin ^{2} \phi \frac{\partial^{2} \psi_{0}}{\partial y^{2}}=0
$$

The boundary conditions (3.6) and (3.7) become

$$
\frac{\partial \psi_{0}}{\partial y}=0, \quad \frac{\partial \psi_{0}}{\partial x}=\cos (x-t), \quad \text { on } y=0,
$$

combined with the requirement that the derivatives of $\psi_{0}$ are bounded as $y \rightarrow \infty$.

Equations (3.10) and (3.11) are solved with the ansatz

$$
\begin{aligned}
& \psi_{0}=f_{1}(y) \cos (x-t)+f_{2}(y) \sin (x-t), \\
& \theta_{0}=g_{1}(y) \cos (x-t)+g_{2}(y) \sin (x-t),
\end{aligned}
$$

for some functions $f_{1}, f_{2}, g_{1}, g_{2}$. Comparing coefficients of sine and cosine leads to a system of four ordinary differential equations,

$$
\begin{gathered}
\left(1+\frac{1}{4} \mu_{2} \sin ^{2} 2 \phi+\mu_{3}\right)\left(f_{1}^{\prime \prime \prime \prime}-2 f_{1}^{\prime \prime}+f_{1}\right)+\mu_{1}\left(\cos 2 \phi\left(g_{1}+g_{1}^{\prime \prime}\right)-2 \sin 2 \phi g_{1}^{\prime}\right) \\
+\mu_{2}\left(\frac{1}{2} \sin 4 \phi\left(f_{2}^{\prime \prime \prime}+f_{2}^{\prime \prime}\right)-\cos 4 \phi f_{1}^{\prime \prime}\right)=0, \\
\left(1+\frac{1}{4} \mu_{2} \sin ^{2} 2 \phi+\mu_{3}\right)\left(f_{2}^{\prime \prime \prime \prime}-2 f_{2}^{\prime \prime}+f_{2}\right)+\mu_{1}\left(\cos 2 \phi\left(g_{2}+g_{2}^{\prime \prime}\right)+2 \sin 2 \phi g_{1}^{\prime}\right) \\
-\mu_{2}\left(\frac{1}{2} \sin 4 \phi\left(f_{1}^{\prime \prime \prime}+f_{1}^{\prime \prime}\right)+\cos 4 \phi f_{2}^{\prime \prime}\right)=0, \\
g_{1}-\sin 2 \phi f_{1}^{\prime}+\sin ^{2} \phi f_{2}^{\prime \prime}-\cos ^{2} \phi f_{2}=0, \\
g_{2}-\sin 2 \phi f_{2}^{\prime}-\sin ^{2} \phi f_{1}^{\prime \prime}+\cos ^{2} \phi f_{1}=0,
\end{gathered}
$$

where the prime denotes differentiation with respect to $y$.

Substituting (3.17) and (3.18) into (3.15) and (3.16), the system reduces to two ordinary differential equations for $f_{1}$ and $f_{2}$. Assuming a basis of solutions of the form

$$
\left(\begin{array}{l}
f_{1} \\
f_{2}
\end{array}\right)=\left(\begin{array}{l}
f_{1}^{0} \\
f_{2}^{0}
\end{array}\right) \mathrm{e}^{\lambda y}
$$

reduces the problem to the linear system

$$
\boldsymbol{L}\left(\begin{array}{l}
f_{1}^{0} \\
f_{2}^{0}
\end{array}\right)=\left(\begin{array}{l}
0 \\
0
\end{array}\right), \quad \text { where } \boldsymbol{L}=\left(\begin{array}{ll}
L_{11} & L_{12} \\
L_{21} & L_{22}
\end{array}\right) .
$$


The components of $L$ are given in appendix B. It should be noted that $L_{11}=L_{22}$ and further that $L_{12}=-L_{21}$. For a non-trivial solution, the determinant of the matrix $L$ must be zero, yielding the equation

$$
L_{11}^{2}+L_{12}^{2}=0
$$

hence,

$$
L_{11}= \pm \mathrm{i} L_{12}
$$

and so

$$
\begin{aligned}
f_{2}^{0} & =-\frac{L_{11}}{L_{12}} f_{1}^{0}, \\
& =\mp \mathrm{i} f_{1}^{0} .
\end{aligned}
$$

Equation (3.21) has eight complex roots, $\lambda_{j}$, four with positive real part and four with negative real part. Since the velocity must remain bounded as $y \rightarrow \infty$, we disregard the positive roots. The other four form two complex conjugate pairs,

$$
\begin{array}{ll}
\lambda_{1}=\alpha_{1}+\mathrm{i} \beta_{1}, & \lambda_{3}=\alpha_{1}-\mathrm{i} \beta_{1}, \\
\lambda_{2}=\alpha_{2}+\mathrm{i} \beta_{2}, & \lambda_{4}=\alpha_{2}-\mathrm{i} \beta_{2} .
\end{array}
$$

It should be noted that the $\lambda_{j}$ are known analytically; however, they are not given here due to space constraints.

The solution form for $\psi_{0}$ is thus

$$
\psi_{0}=\sum_{j=1}^{4} \hat{A_{j}}\left(\cos (x-t)+\xi_{j} \sin (x-t)\right) \mathrm{e}^{\lambda_{j} y},
$$

where $\xi_{j}=-\mathrm{i}$ for $j=1,2$ and $\xi_{j}=\mathrm{i}$ for $j=3,4$. Assuming that the constants take the general form $\hat{A}_{j}=A_{j}+\mathrm{i} B_{j}$ for $j=1,2,3,4$, the boundary conditions (3.12) give

$$
\begin{array}{cc}
A_{1}=\frac{\alpha_{1} \beta_{2}-\alpha_{2} \beta_{1}}{2\left(\left(\alpha_{1}-\alpha_{2}\right)^{2}+\left(\beta_{1}-\beta_{2}\right)^{2}\right)}, \quad A_{2}=-A_{1}, & A_{3}=A_{1}, \quad A_{4}=-A_{1}, \\
B_{1}=\frac{\alpha_{2}^{2}-\alpha_{1} \alpha_{2}+\beta_{2}^{2}-\beta_{1} \beta_{2}}{2\left(\left(\alpha_{1}-\alpha_{2}\right)^{2}+\left(\beta_{1}-\beta_{2}\right)^{2}\right)}, & B_{3}=-B_{1}, \\
B_{2}=\frac{\alpha_{1}^{2}-\alpha_{1} \alpha_{2}+\beta_{1}^{2}-\beta_{1} \beta_{2}}{2\left(\left(\alpha_{1}-\alpha_{2}\right)^{2}+\left(\beta_{1}-\beta_{2}\right)^{2}\right)}, & B_{4}=-B_{2} .
\end{array}
$$

The fibre angle perturbation is then of the form

$$
\begin{aligned}
\theta_{0}= & \sum_{j=1}^{4} \hat{A}_{j}\left[\left(\lambda_{j} \sin 2 \phi+\xi_{j}\left(-\lambda_{j}^{2} \sin ^{2} \phi+\cos ^{2} \phi\right)\right) \cos (x-t)\right. \\
& \left.+\left(\xi_{j} \lambda_{j} \sin 2 \phi+\lambda_{j}^{2} \sin ^{2} \phi-\cos ^{2} \phi\right) \sin (x-t)\right] \mathrm{e}^{\lambda_{j} y} .
\end{aligned}
$$

The change in the small perturbation to the orientation, $\theta_{0}$, is dependent on the initial orientation angle of the fibres along with their position. 


\subsection{Order- $\varepsilon^{2}$ solution and mean swimming velocity}

The mean swimming velocity is determined by the horizontal component of the flow as $y$ tends to infinity. The leading-order stream function, equation (3.26), tends to zero, and hence the non-zero mean swimming velocity is determined at order $\varepsilon^{2}$,

$$
U \sim \varepsilon^{2} U_{1}=\lim _{y \rightarrow \infty} \varepsilon^{2} \frac{\partial \psi_{1}}{\partial y}
$$

We neglect the oscillatory terms to determine the leading-order term in the expansion of mean swimming velocity, which we denote as $\bar{U}_{1}$. The bar notation represents an average over one time period.

At order $\varepsilon^{2}$, the boundary conditions (3.6) and (3.7) become

$$
\left.\frac{\partial \psi_{1}}{\partial y}\right|_{y=0}+\left.\sin (x-t) \frac{\partial^{2} \psi_{0}}{\partial y^{2}}\right|_{y=0}=0,\left.\quad \frac{\partial \psi_{1}}{\partial x}\right|_{y=0}+\left.\sin (x-t) \frac{\partial^{2} \psi_{0}}{\partial y \partial x}\right|_{y=0}=0, \quad(3.30 a, b)
$$

and hence

$$
\begin{gathered}
\left.\frac{\partial \psi_{1}}{\partial y}\right|_{y=0}=\frac{1}{2}\left(\left(\alpha_{1} \alpha_{2}-\beta_{1} \beta_{2}\right)(1-\cos 2(x-t))-\left(\alpha_{1} \beta_{2}-\alpha_{2} \beta_{1}\right) \sin 2(x-t)\right), \\
\left.\frac{\partial \psi_{1}}{\partial x}\right|_{y=0}=0 .
\end{gathered}
$$

Because of the form of the boundary conditions, the ansatz is

$$
\psi_{1}=\hat{f}_{1}(y)+\hat{f}_{2}(y) \cos 2(x-t)+\hat{f}_{3}(y) \sin 2(x-t),
$$

for some functions $\hat{f}_{1}, \hat{f}_{2}$ and $\hat{f}_{3}$. Substituting the solution form (3.33) into the order- $\varepsilon^{2}$ expansion of (3.3), shown in full in appendix B, and equating coefficients of nonoscillating terms, we have

$$
\hat{f}_{1}^{\prime \prime \prime \prime}=0
$$

and hence

$$
\hat{f}_{1}(y)=A y^{3}+B y^{2}+C y+D
$$

To ensure that the velocity remains bounded as $y \rightarrow \infty$, we set $A=B=0$. From the boundary conditions (3.31) and (3.32), we find that $C=\left(\alpha_{1} \alpha_{2}-\beta_{1} \beta_{2}\right) / 2$ and $D=0$, and hence

$$
\hat{f}_{1}(y)=\frac{y}{2}\left(\alpha_{1} \alpha_{2}-\beta_{1} \beta_{2}\right)
$$

Differentiating $\hat{f}_{1}(y)$ with respect to $y$, the leading-order term in the expansion of mean swimming velocity is calculated as

$$
\bar{U}_{1}=\frac{1}{2}\left(\alpha_{1} \alpha_{2}-\beta_{1} \beta_{2}\right)
$$




\subsection{Mean rate of working}

To determine how Stokesian swimming is affected by transverse isotropy, the mean rate of working at order $\varepsilon$ is investigated, i.e. the rate of working per unit area of the sheet against viscous stress, $\varepsilon^{2} \bar{W}$ (Taylor 1951). The mean value of this quantity is given by

$$
\varepsilon^{2} \bar{W}=-\overline{\left.\frac{\partial y_{s}}{\partial t} \sigma_{22}\right|_{y=0}},
$$

where $y_{s}$ is the equation of the sheet surface and $\left.\sigma_{22}\right|_{y=0}$ is the normal stress evaluated on the sheet. The no-slip condition is $u=0$ on the sheet, and hence $\partial u / \partial x=0$, and, via (2.8), $\partial v / \partial y=0$. In terms of the stream function, $\sigma_{22}$ is

$$
\sigma_{22}=-p+\mu_{1} \sin ^{2} \phi+\left(\mu_{2} \cos \phi \sin ^{3} \phi+\mu_{3} \sin 2 \phi\right)\left(\frac{\partial^{2} \psi}{\partial y^{2}}-\frac{\partial^{2} \psi}{\partial x^{2}}\right) .
$$

Solving (2.9), using the leading-order expression for the stream function (3.26), determines the pressure. Noting that $\overline{\cos ^{2}(x-t)}=1 / 2$, we obtain an expression for the leading-order term in the expansion of the mean rate of working,

$$
\begin{aligned}
\bar{W}= & -\frac{1}{16}\left[\left(\alpha_{1}^{2} \alpha_{2}-\alpha_{2} \beta_{1}\left(\beta_{1}+2 \beta_{2}\right)\right.\right. \\
& \left.+\alpha_{1}\left(\alpha_{2}^{2}-\beta_{2}\left(2 \beta_{1}+\beta_{2}\right)\right)\right)\left(8+\mu_{2}(1+\cos 4 \phi)+8 \mu_{3}\right) \\
& \left.+4\left(\alpha_{2} \beta_{1}+\alpha_{1} \beta_{2}\right) \mu_{2} \sin 4 \phi\right] .
\end{aligned}
$$

\section{Results}

The leading-order terms in the expansions of the mean swimming velocity (3.37), the mean rate of working (3.40), the fibre perturbation (3.28), the velocities $u_{0}=$ $\partial \psi_{0} / \partial y, v_{0}=-\partial \psi_{0} / \partial x$ and the stream function (3.26) have been found analytically in terms of lengthy expressions for $\alpha_{j}, \beta_{j}$. The analytical results for the mean rate of working have been recreated numerically using finite differences and integration by the midpoint method. Each separate component has been verified along with the full solution. The solutions agreed to within a small degree of numerical error. For brevity, we will refer to the time averages of the leading-order terms in the expressions for the swimming velocity and the rate of working as the mean swimming velocity and the mean rate of working respectively, and we will plot terms without the leading $\varepsilon^{2}$ factors as defined by $\bar{U}_{1}, \bar{W}$ in (3.29) and (3.38).

We now discuss the results in more detail. Four different flow regimes are considered (figure 3): (i) a passive transversely isotropic fluid, occurring when $\mu_{1}=0$; (ii) an active fluid where $\mu_{2}=\mu_{3}=0$ and $\mu_{1}$ is non-zero; (iii) a nearly isotropic regime, where all parameters take values up to 5; (iv) the regime where at least one of $\mu_{1}, \mu_{2}$ and $\mu_{3}$ is much larger than 1. A range of initial orientation angles, $\phi$, between 0 and $2 \pi$ is considered for all regimes and the active parameter, $\mu_{1}$, is allowed to take both positive and negative values to account for 'puller' and 'pusher' active behaviour respectively. It should be noted that since the fibres have no directionality, the regime $\phi=0-\pi$ is identical to $\phi=\pi-2 \pi$.

\subsection{Regime (i): effect of passive fibres on mean swimming velocity and rate of working}

Passive fibres exert no shear-independent force and have no self-propulsive properties; hence, the active parameter $\mu_{1}$ is set to zero. In this regime, the mean swimming 

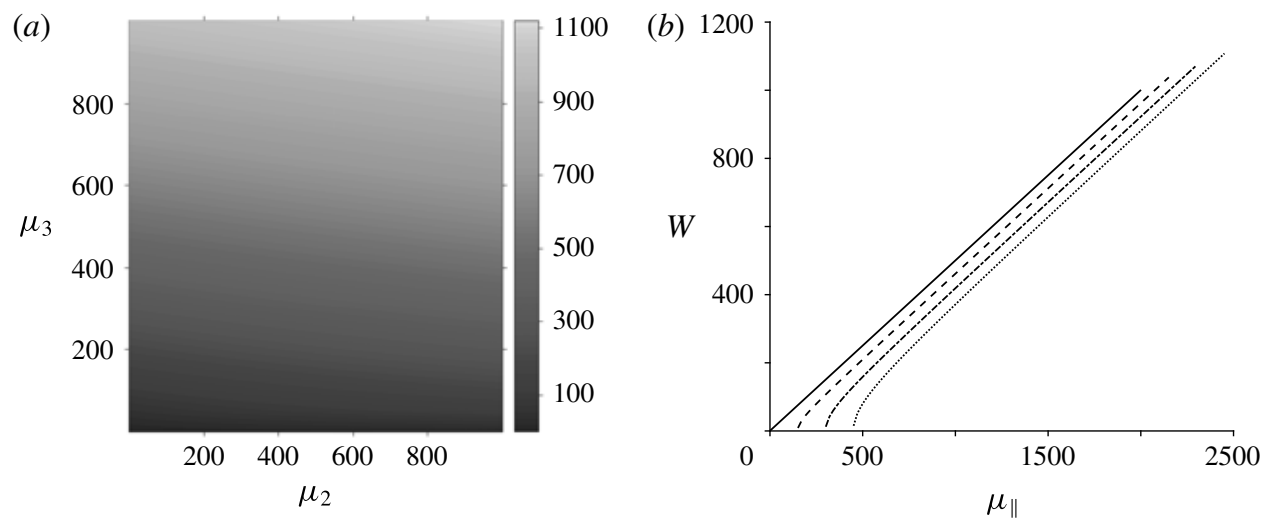

FIGURE 5. Mean rate of working by the swimmer in a transversely isotropic fluid where $\mu_{1}=0$. (a) The mean rate of working for varying $\mu_{2}$ and $\mu_{3}$. This result is identical for all initial angles $\phi$. (b) The change in the mean rate of working for increasing $\mu_{\|}$for a range of $\mu_{3}$ and set $\mu_{2}$ values: $\mu_{2}=0$ (solid line), $\mu_{2}=300$ (dashed line), $\mu_{2}=600$ (dash-dotted line) and $\mu_{2}=900$ (dotted line).

velocity takes on the Newtonian value, $\bar{U}_{1}=1 / 2$, and the mean rate of working is independent of the initial orientation angle $\phi$. In figure $5(a)$, the mean rate of working is always greater than or equal to the Newtonian case, $\bar{W}=1$. The increase in the mean rate of working is linear throughout, with $\mu_{3}$ having a larger impact than $\mu_{2}$. Figure $5(b)$ depicts the relationship between the mean rate of working and the parallel viscosity $\mu_{\|}=1+\left(\mu_{2}+4 \mu_{3}\right) / 2$, where each line represents a different value of $\mu_{2}$. The increase in the mean rate of working with $\mu_{\|}$is linear, apart from the case where $\mu_{3}$ is small and $\mu_{2}$ is large, with a large mean rate of working as $\mu_{2}$ increases.

\subsection{Regime (ii): active-only effects on mean swimming velocity and rate of working}

The active-only regime considers $\mu_{2}$ and $\mu_{3}$ to be zero with $\mu_{1}$ non-zero. The mean swimming velocity is considered in figure 6 and the mean rate of working in figure 7 . For $\mu_{1}=0$, we regain the Newtonian result, and hence both the mean swimming velocity and the mean rate of working are independent of the fibre angle. For non-zero active parameter $\mu_{1}$, the mean swimming velocity and mean rate of working vary considerably with the fibre angle. In particular, negative mean swimming velocity i.e. reversal of swimming direction - and negative mean rate of working are observed in certain regimes for large $\mu_{1}$, with a sudden and dramatic switch in sign close to $\phi=3 \pi / 4$ (figures $6 c, d$ and $7 c, d$ ). It should be noted that this can be resolved through refinement of the plotting grid and is not a discontinuity. A change from 'pusher'- to 'puller'-type active behaviour (equivalent to a change in sign of $\mu_{1}$ ) is equivalent to a reflection in the line $\phi=\pi / 2(3 \pi / 2)$.

\subsection{Regime (iii): nearly isotropic behaviour in leading-order mean swimming velocity and rate of working}

A small perturbation away from the isotropic case is considered here; $\mu_{1}, \mu_{2}$ and $\mu_{3}$ take values of up to 5 . When $\mu_{1}$ is much smaller than 1 and positive (figure $8 a, b$ ), a small perturbation away from the Newtonian case is observed. As $\mu_{1}$ continues to increase, angular dependence becomes more prevalent. For the mean swimming 

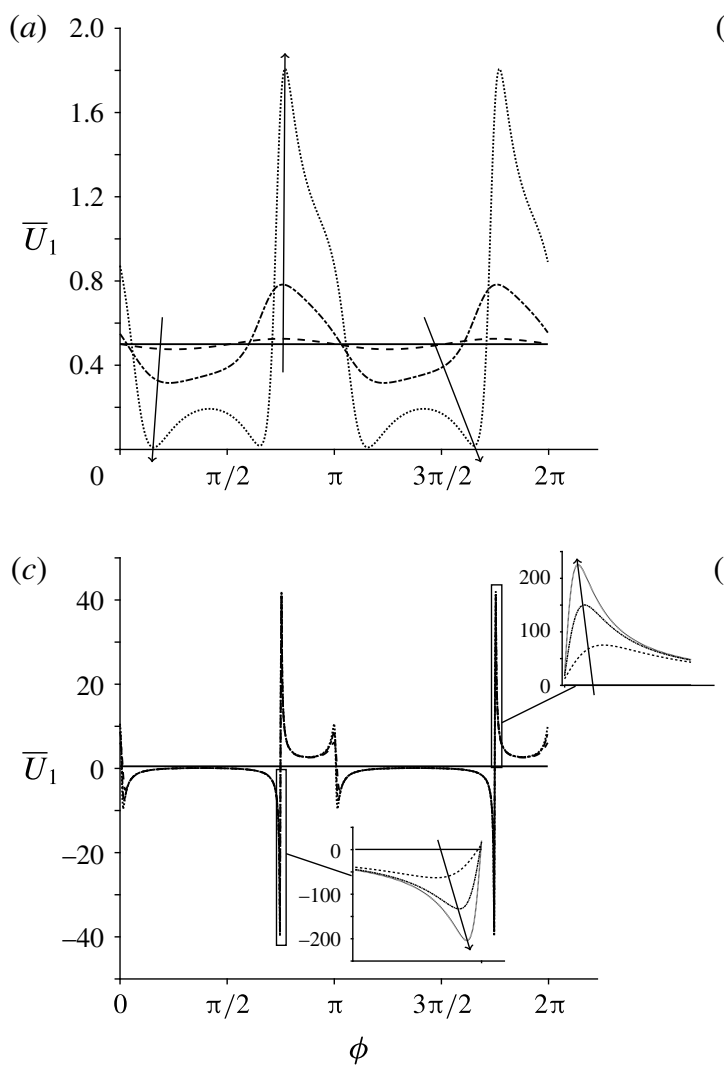

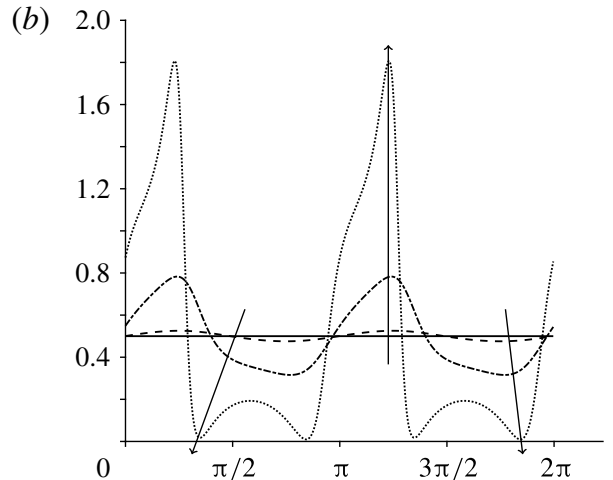

(d)

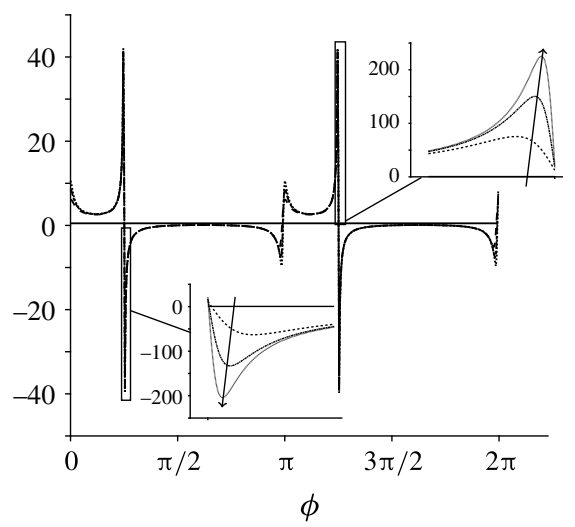

FIGURE 6. Mean swimming velocity versus the initial orientation $\phi$, where $\mu_{2}=\mu_{3}=0$. Panels $(a)$ and $(b)$ depict small positive and negative $\mu_{1}$ values: 0 (solid line), \pm 0.1 (dashed line), \pm 1 (dash-dotted line) and \pm 5 (dotted line). Panels $(c)$ and $(d)$ depict larger $\mu_{1}$ values: 0 (solid line), \pm 300 (dashed line), \pm 600 (dash-dotted line), \pm 900 (dotted line), where the arrows denote increasing $\mu_{1}$.

velocity, $\mu_{2}$ has minimal impact, while $\mu_{3}$ reduces the range of values that the background flow can take. For the mean rate of working (figure 9), $\mu_{2}$ again has little impact on the results, and the effect of increasing $\mu_{3}$ is to increase the cost of swimming. When $\mu_{1}= \pm 5$ (figure $9 e, f$ ), the mean rate of working may become negative, and the effect of increasing $\mu_{3}$ is to reduce the range of values that the mean rate of working will take.

\subsection{Regime (iv): the effect of large rheological parameters on leading-order mean swimming velocity and rate of working}

The final regime is where at least one of $\mu_{1}, \mu_{2}$ and $\mu_{3}$ is much larger than 1 . Figures 10 and 11 depict how the mean swimming velocity and mean rate of working change with the initial orientation angle, $\phi$. When either $\mu_{2}$ or $\mu_{3}$ is non-zero, the

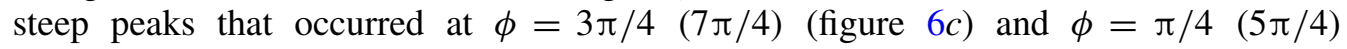
(figure $6 d$ ) within regime (ii) no longer appear. Further, when $\mu_{2}$ is non-zero and $\mu_{3}=0$, the mean swimming velocity becomes negative for certain initial orientation angles, i.e. the swimming direction is reversed. When $\mu_{3}$ becomes non-zero, the 

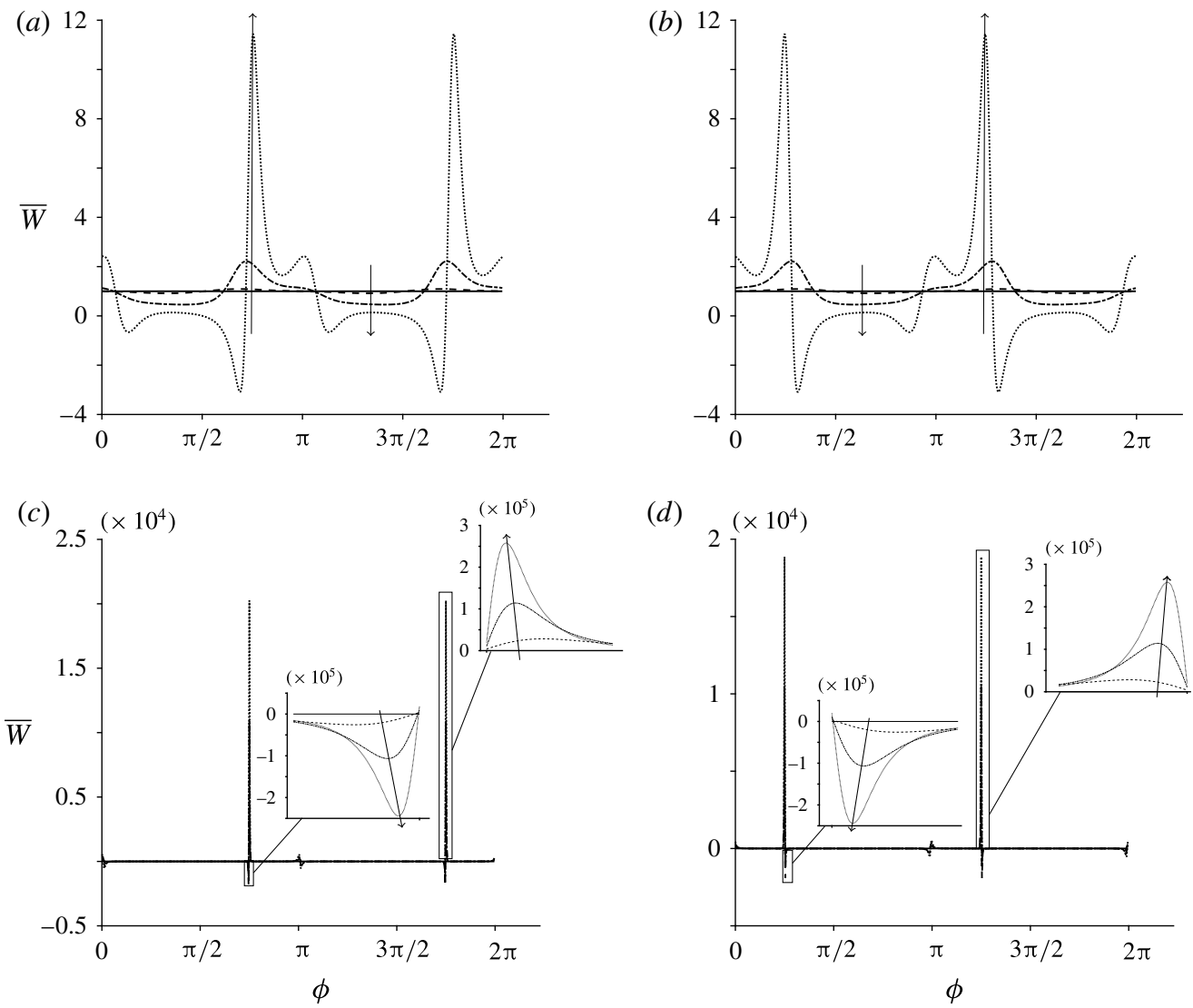

FIgURE 7. Mean rate of working versus the initial orientation $\phi$, where $\mu_{2}=\mu_{3}=0$. Panels $(a)$ and $(b)$ depict small positive and negative $\mu_{1}$ values: 0 (solid line), \pm 0.1 (dashed line), \pm 1 (dash-dotted line) and \pm 5 (dotted line). Panels $(c)$ and $(d)$ depict larger $\mu_{1}$ values: 0 (solid line), \pm 300 (dashed line), \pm 600 (dash-dotted line), \pm 900 (dotted line), where the arrows denote increasing $\mu_{1}$.

results collapse down towards the Newtonian case, altered predominantly by the active parameter, $\mu_{1}$. Similar results are seen for the mean rate of working; however, for non-zero $\mu_{3}$ (figure $11 c, d$ ), the reference value about which variations occur is significantly increased.

\subsection{Orientation, velocity and stream function}

Finally, to understand how the fibre orientation and velocity are impacted by the anisotropic fluid properties, the orientation angle $(\phi+\theta)$ and velocity are considered in active and passive regimes, and the stream function is considered in all four regimes of interest (figures 12-14 respectively). Each variable is plotted for one wavelength of the sheet, $x=0-2 \pi$. We focus on the case where the fibres are aligned with the sheet, i.e. $\phi=0$, and plot results at time $t=0$ (i.e. the start of one oscillation period). See the supplementary movies available at https://doi.org/10.1017/jfm.2016.821.

Considering first the fibre orientation, in all cases, perturbations to the initial orientation angle are greater in the vicinity of the sheet and are displaced with the 

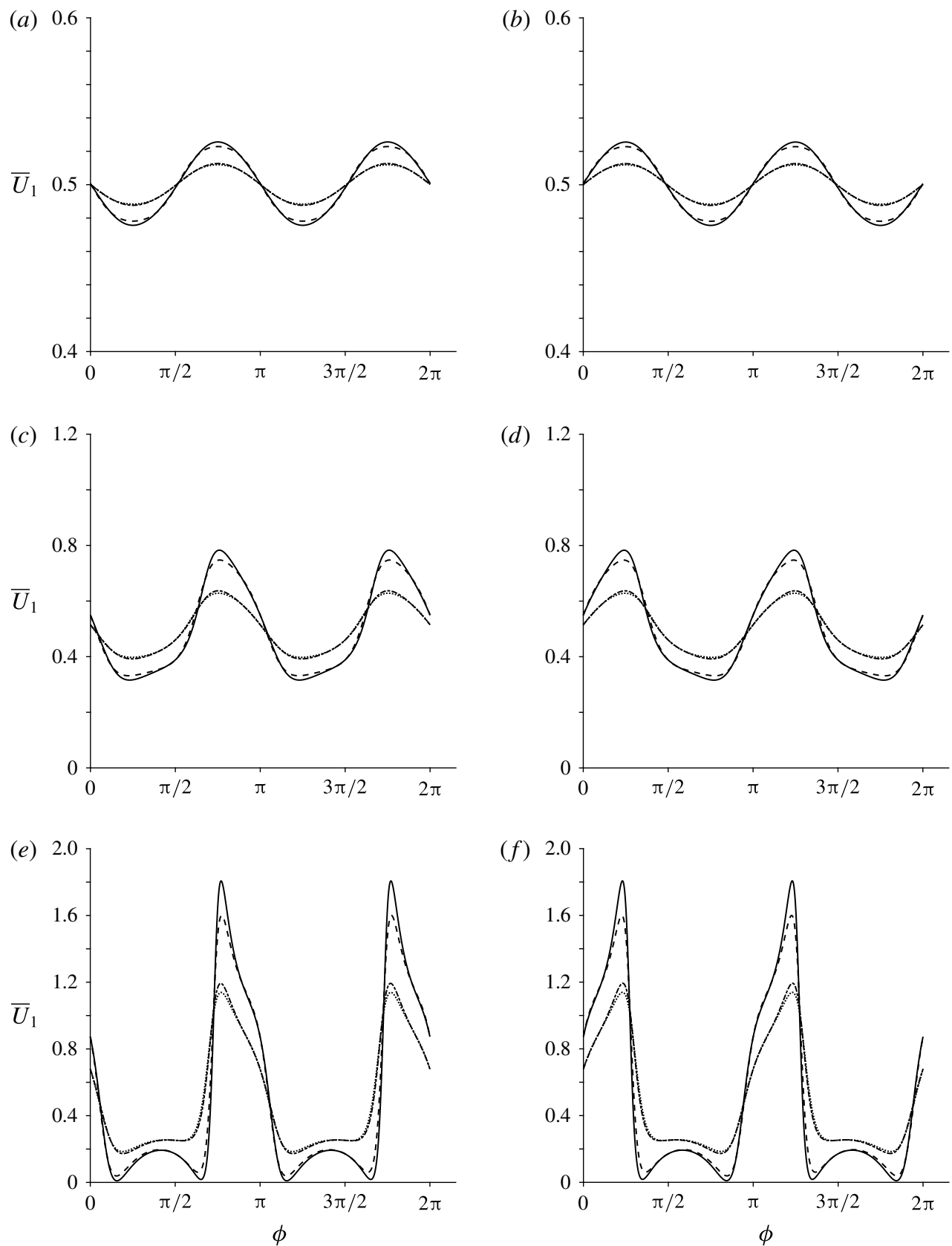

FIGURE 8. Mean swimming velocity versus $\phi$, where the parameters $\mu_{1}, \mu_{2}$ and $\mu_{3}$ take values of up to 5. Panels $(a)$ and $(b)$ show $\mu_{1}= \pm 0.1,(c)$ and $(d)$ show $\mu_{1}= \pm 1$ and (e) and $(f)$ show $\mu_{1}= \pm 5$. Each line depicts a different $\mu_{2}$ and $\mu_{3}$ combination: $\mu_{2}=$ $0, \mu_{3}=0$ (solid line), $\mu_{2}=1, \mu_{3}=0$ (dashed line), $\mu_{2}=0, \mu_{3}=1$ (dash-dotted line) and $\mu_{2}=1, \mu_{3}=1$ (dotted line). 

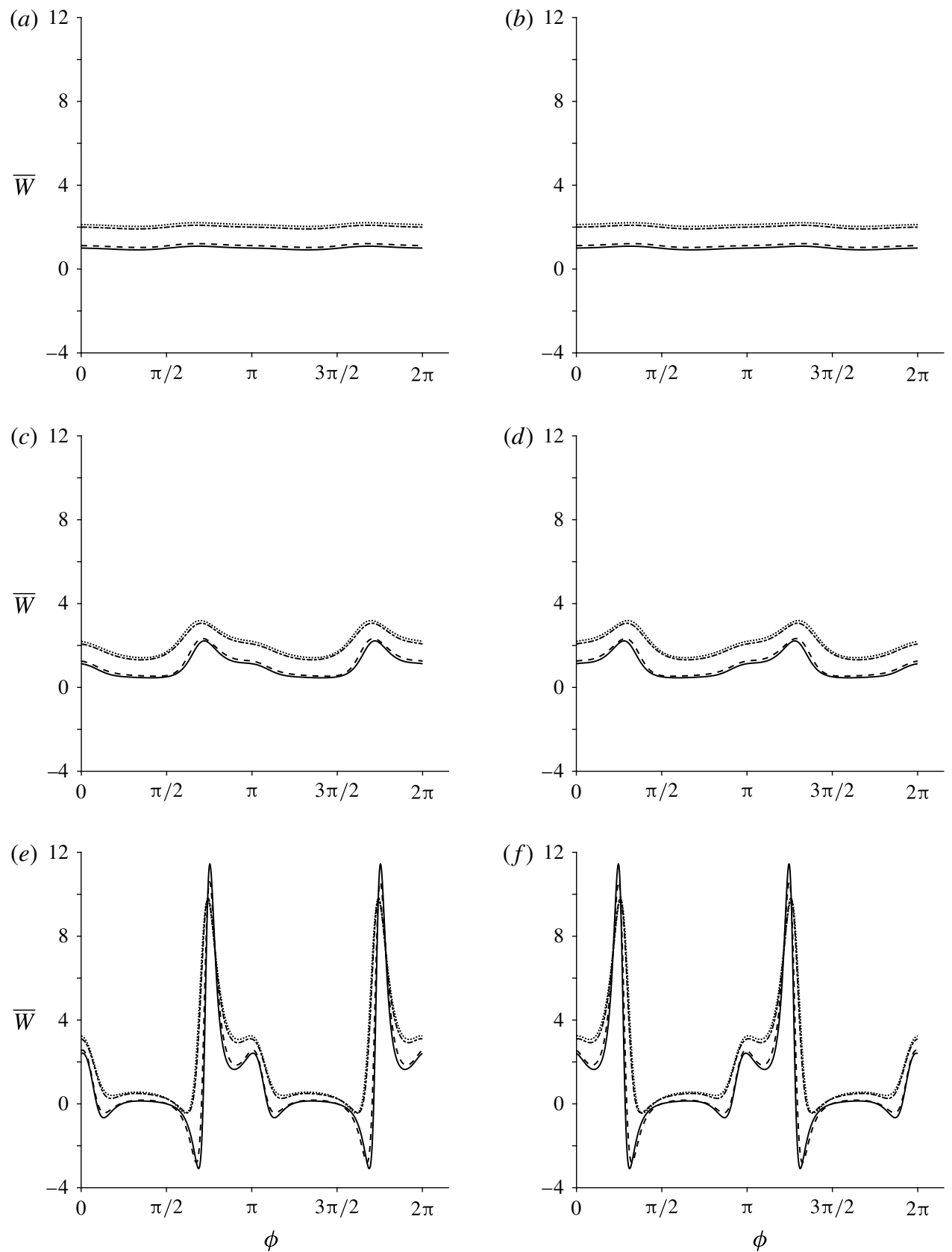

FIGURE 9. Mean rate of working versus $\phi$, where the parameters $\mu_{1}, \mu_{2}$ and $\mu_{3}$ take values of up to 5. Panels $(a)$ and $(b)$ show $\mu_{1}= \pm 0.1,(c)$ and $(d)$ show $\mu_{1}= \pm 1$ and (e) and $(f)$ show $\mu_{1}= \pm 5$. Each line depicts a different $\mu_{2}$ and $\mu_{3}$ combination: $\mu_{2}=$ $0, \mu_{3}=0$ (solid line), $\mu_{2}=1, \mu_{3}=0$ (dashed line), $\mu_{2}=0, \mu_{3}=1$ (dash-dotted line) and $\mu_{2}=1, \mu_{3}=1$ (dotted line).

movement of the sheet (figure 12 and movie 1). For passive rheology, the fibre reorientation is dampened very quickly moving away from the sheet (figure 12a). 

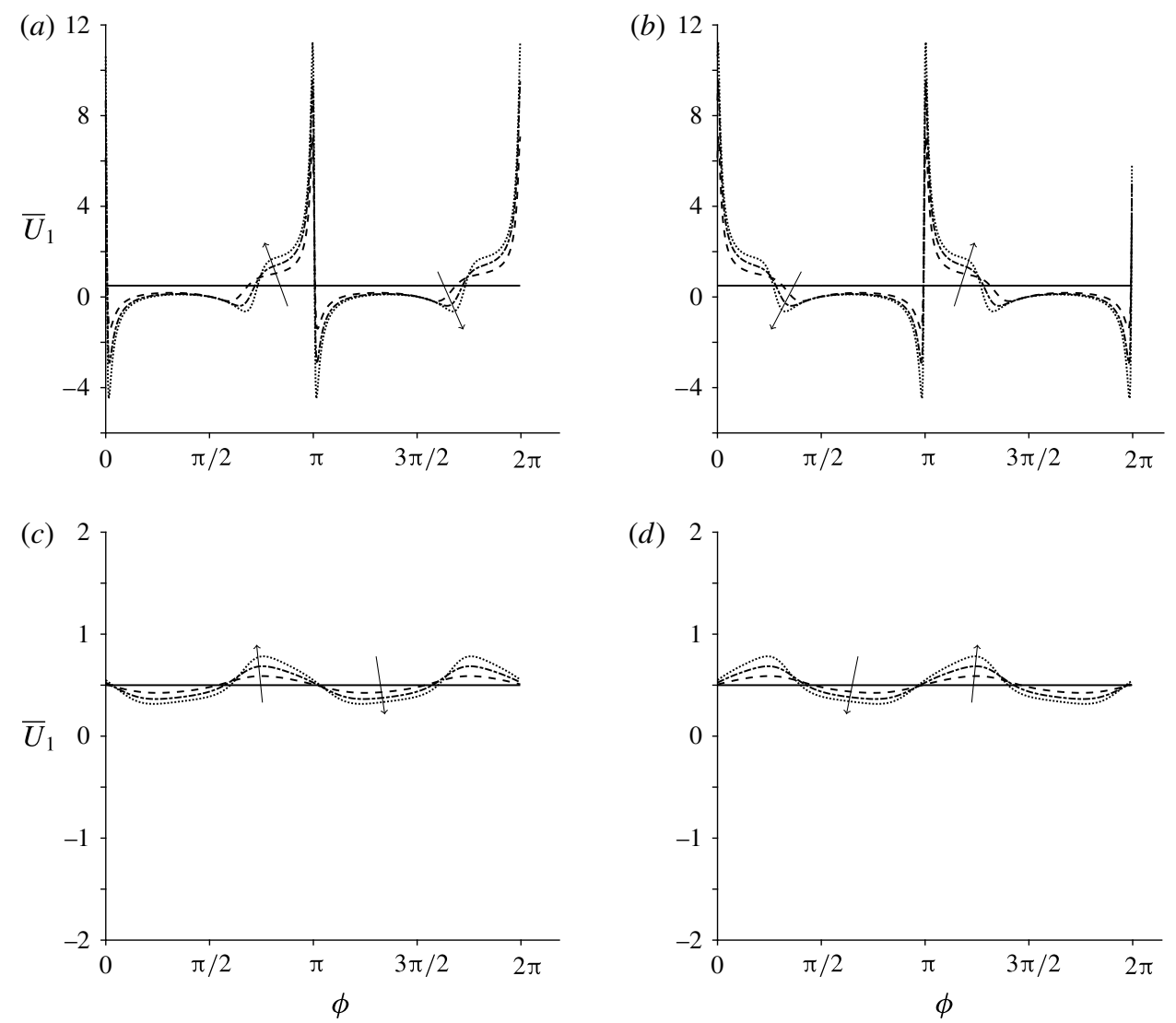

FIGURE 10. Mean swimming velocity versus $\phi$ when at least one of $\mu_{1}, \mu_{2}$ and $\mu_{3}$ is much larger than 1. Panels $(a)$ and $(c)$ depict positive $\mu_{1}$ values and $(b)$ and $(d)$ depict negative $\mu_{1}$ values. The values $\mu_{1}$ takes are 0 (solid line), \pm 300 (dashed line), \pm 600 (dash-dotted line) and \pm 900 (dotted line). In $(a)$ and $(b), \mu_{2}=900, \mu_{3}=0$, and in $(c)$ and $(d), \mu_{2}=0, \mu_{3}=900$, where the arrows denote increasing $\mu_{1}$.

Once $\mu_{1}$ is non-zero, fibre displacement appears further away from the sheet (figure $12 b$ ) and movements propagate to the right.

Figure 13 and movie 2 show the velocity components in $x$ and $y$. The leading-order velocity decays quickly moving away from the sheet, as is evident in figure 13(a); in the active-only regime (figure 13b), the flow decays more slowly. The velocity field shows a similar rightward propagation to fibre angle in the active-fluid case (figures $12 b$ and 13b). These results are mirrored in figure 14 and movie 3, where the streamlines of the resulting flow are displayed. In the passive regime (i), the streamlines are symmetric about $x=\pi$ with anticlockwise flow between $x=0$ and $\pi$ and clockwise flow for $x=\pi-2 \pi$. Introducing $\mu_{1}$ distorts the streamlines, and, when $\mu_{2}=\mu_{3}=0$, the streamlines are deflected to the right (figure 14b); introducing the other two parameters dampens this deflection (figure $14 c, d$ ).

\section{Discussion}

The classical Taylor's swimming sheet problem was modified to account for transverse isotropy, modelling swimming in fibre-reinforced fluids or active media. 

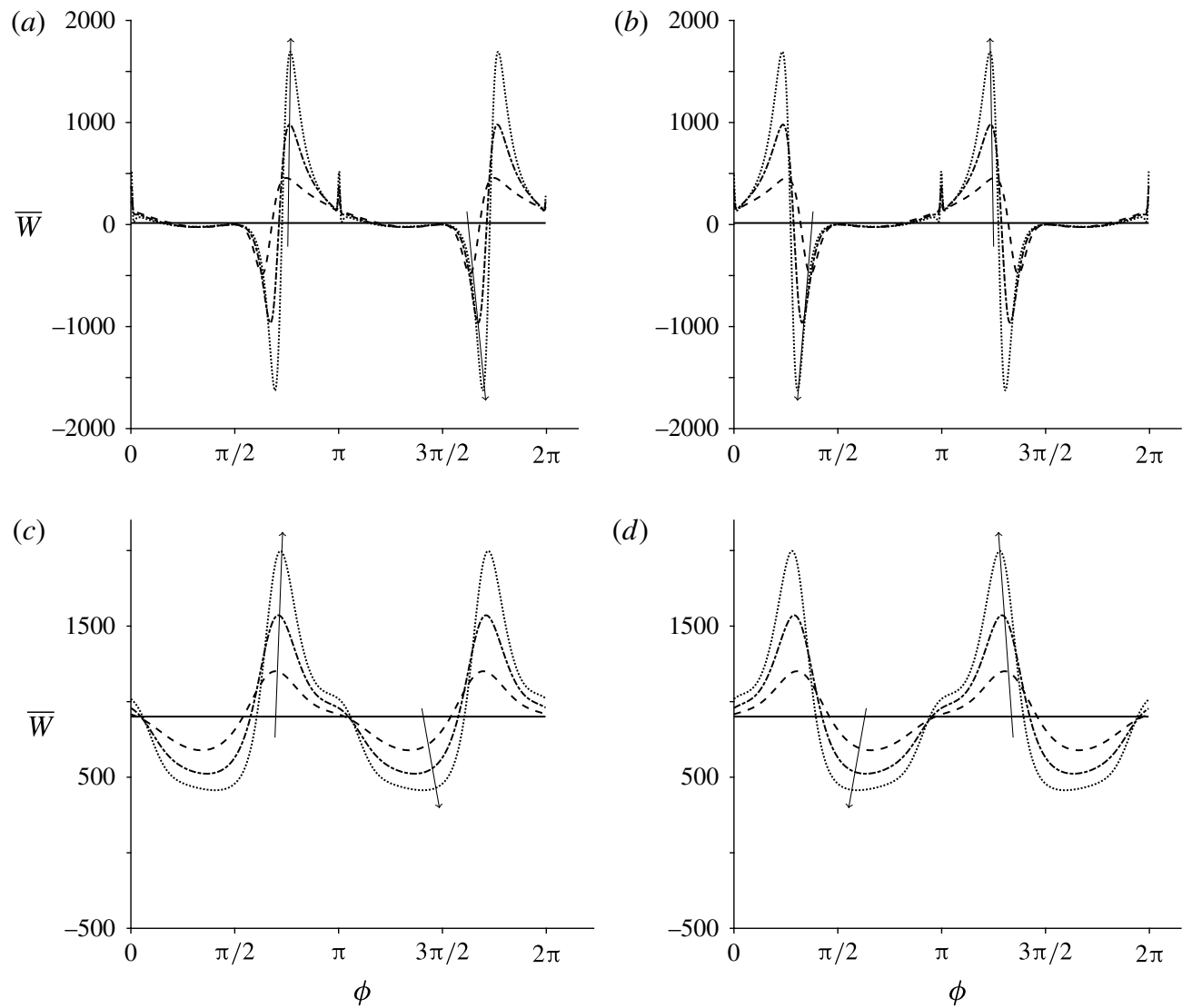

FIgURE 11. Mean rate of working versus $\phi$ when at least one of $\mu_{1}, \mu_{2}$ and $\mu_{3}$ is much larger than 1. Panels $(a)$ and $(c)$ depict positive $\mu_{1}$ values and $(b)$ and $(d)$ depict negative $\mu_{1}$ values. The values $\mu_{1}$ takes are 0 (solid line), \pm 300 (dashed line), \pm 600 (dash-dotted line) and \pm 900 (dotted line). In $(a)$ and $(b), \mu_{2}=900, \mu_{3}=0$, and in $(c)$ and $(d), \mu_{2}=$ $0, \mu_{3}=900$, where the arrows denote increasing $\mu_{1}$.

The quantities of interest were the steady background flow, which corresponds to the mean swimming velocity, and the mean rate of working. The results presented were non-dimensional. The dimensional velocity scales with the wave speed, and the rate of working scales with the square of the frequency, the viscosity and the wavenumber. The ratio of the mean swimming speed to the wave speed is proportional to $1 / \varepsilon^{2}$. When $\bar{U}_{1}$ takes the maximum value found here, such that $\bar{U}_{1} \approx 40$, this corresponds to swimming faster than the wave speed when $\varepsilon>1 / \sqrt{\bar{U}_{1}} \approx 0.16$. It should be noted that swimming with a prescribed beat amplitude and frequency, regardless of the rheology of the fluid, will not in general be achievable in a real biological system.

When $\mu_{1}$ is zero, no dependence on the initial orientation angle is observed and the mean swimming velocity takes on the Newtonian value, regardless of the size of the other parameters. For non-zero $\mu_{1}$, certain initial orientation angles enable less energetically costly but slower swimming, with lower mean rates of working and swimming velocities. The initial angles maximising the mean swimming velocity and the mean rate of working are not in general the same. When active fibres are parallel to the swimming direction, swimming is both faster and more energetically 
(a)

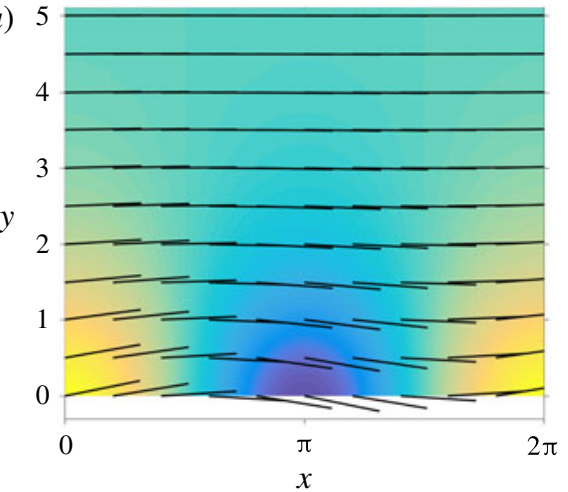

(b)

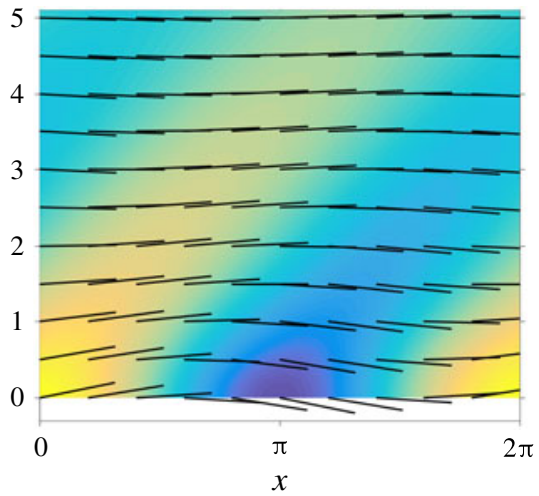

FIGURE 12. Fibre angle, $\phi+\varepsilon \theta_{0}$, in passive and active regimes: $(a)$ the passive regime $\left(\mu_{1}=0, \mu_{2}=\mu_{3}=5\right)$ and $(b)$ the active-only regime $\left(\mu_{1}=5, \mu_{2}=\mu_{3}=0\right)$. In each panel, $t=0, \varepsilon=0.2$ and the initial orientation angle is $\phi=0$. See movie 1 .

(a)

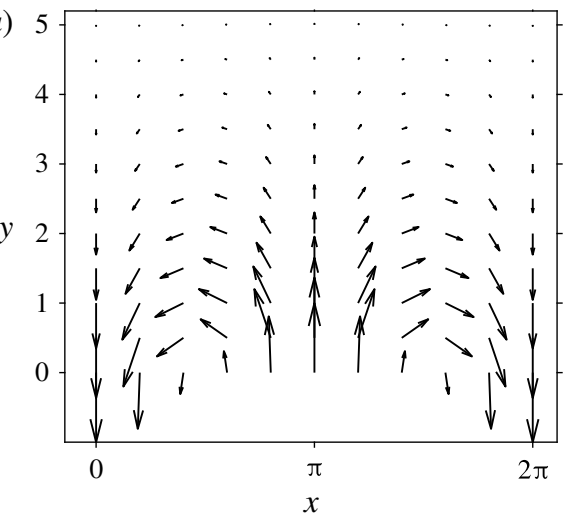

(b)

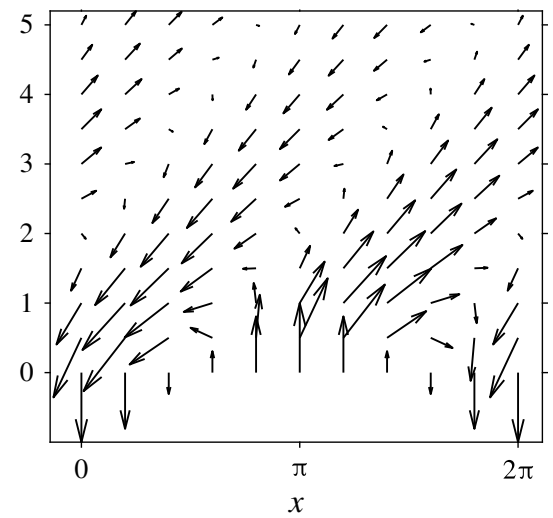

FIGURE 13. Velocity field in passive and active regimes: $(a)$ passive regime $\left(\mu_{1}=0, \mu_{2}=\right.$ $\left.\mu_{3}=5\right)$ and $(b)$ active-only regime $\left(\mu_{1}=5, \mu_{2}=\mu_{3}=0\right)$. In each panel, $t=0$ and the initial orientation angle is $\phi=0$. See movie 2 .

costly compared with active fibres perpendicular to the swimming direction. The sheet utilises the surrounding environment to boost its velocity, a result seen in Leshansky (2009) for point-like obstacles and Chrispell, Fauci \& Shelley (2013) for swimming in viscoelastic fluids near walls. However, these authors also predict an increased swimming efficiency, a result not seen here. A change from 'pusher' to 'puller' behaviour (equivalent to a change in sign of $\mu_{1}$ ) equates to a reflection of the initial fibre angle in the $y$-axis. The activity parameter $\mu_{1}$ allows the mean rate of working to take on negative values, suggesting that the tension/stresslet exerted by the fibres on the sheet may at times overcome the work the sheet does on the fluid to move. For some values of $\mu_{1}$, the mean swimming velocity may be negative, indicating a reversal of swimming direction; this change is dependent on the uniform orientation angle $\phi$, a result also observed for rotated viscoelastic networks (Wróbel et al. 2016). The inclusion of active behaviour dramatically changes the streamlines and flow field. 


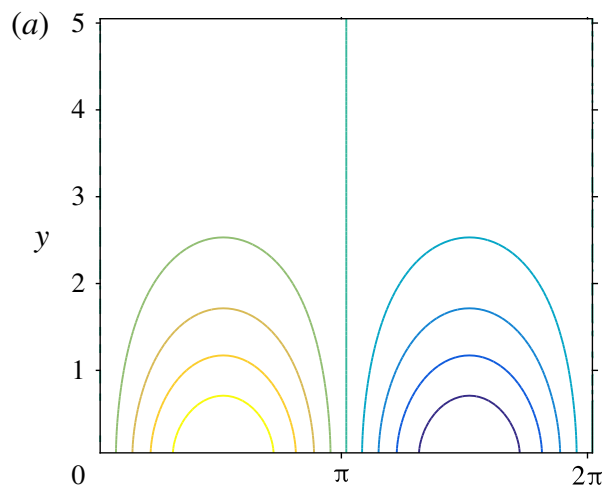

(b)
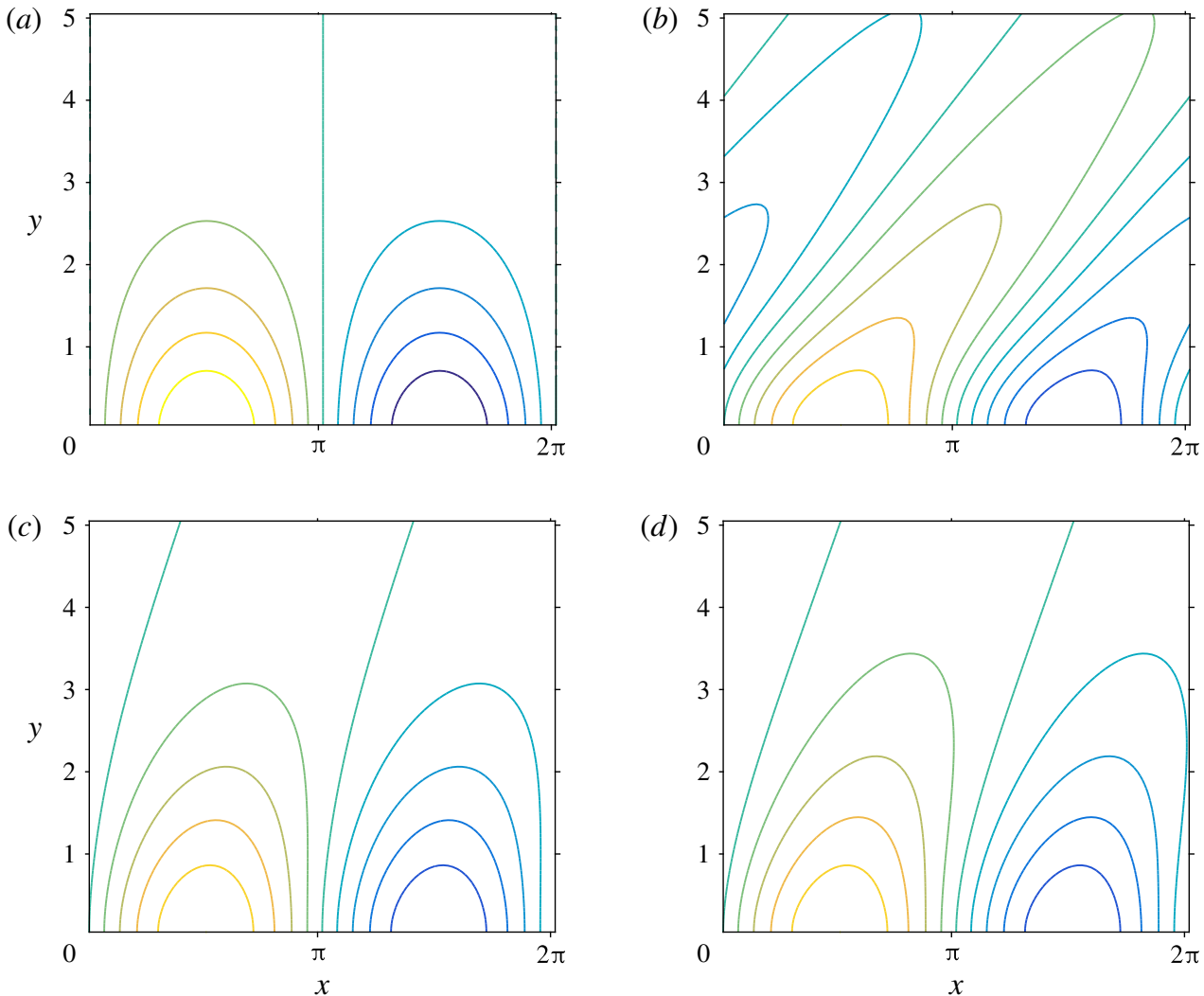

FIGURE 14. Instantaneous streamlines in the four regimes considered: (a) passive regime $\left(\mu_{1}=0, \mu_{2}=\mu_{3}=5\right),(b)$ active-only regime $\left(\mu_{1}=5, \mu_{2}=\mu_{3}=0\right),(c)$ nearly isotropic regime $\left(\mu_{1}=\mu_{3}=1, \mu_{2}=0\right)$ and $(d)$ regime where at least one of the parameters is much larger than $1\left(\mu_{1}=\mu_{2}=\mu_{3}=900\right)$. In each panel, $t=0$ and the initial orientation angle is $\phi=0$. See movie 3 .

For a passive transversely isotropic fluid, i.e. $\mu_{1}=0$, increasing the magnitude of the viscosity-like parameters increases the work the sheet must do on the fluid to swim; the fluid becomes more difficult to move through. The mean rate of working was found to be approximately linear in the parallel viscosity $\mu_{\|}=1+\left(\mu_{2}+4 \mu_{3}\right) / 2$, with a small additional dependence on $\mu_{3}$. For an active isotropic fluid $\left(\mu_{1}\right.$ is non-zero and $\mu_{2}=\mu_{3}=0$ ), we observe potentially unphysical behaviour when $\mu_{1}$ is increased sufficiently, with rapid large variations with respect to $\phi$ in both the mean swimming velocity and the rate of working. It should be noted, however, that a large value of $\mu_{1}$ with $\mu_{2}=\mu_{3}=0$ may not represent a physically realistic fluid. These unphysical effects are reduced by the inclusion of $\mu_{3}$ in particular, and to a lesser extent $\mu_{2}$, dampening these large variations.

When the rheological parameters are all non-zero, increasing the anisotropic shear viscosity causes the mean swimming velocity to collapse down towards the Newtonian result, altered slightly by the active properties of the fluid. However, the mean rate of working is increased in general. The anisotropic extensional viscosity has a similar but much smaller effect. 
This study demonstrates that locomotion in active fluids is dramatically different from locomotion in passive fluids. Our finding of zero, and indeed negative, rate of working for some angular configurations and sufficiently large values of the active parameter $\mu_{1}$ is a consequence of the energy input to the system by active stress. This phenomenon may be related to superfluid behaviour recently observed in bacteria suspensions (López et al. 2015). Further, these results are suggestive that active stresses in the bulk may enhance the motion of individual swimmers. It has long been known that some flagellated swimmers may propel more rapidly in polymeric than Newtonian fluids (Schneider \& Doetsch 1974). An increase in the anisotropy of the slender-body drag coefficients has been proposed as one underlying mechanism (Berg \& Turner 1979; Magariyama \& Kudo 2002); recently, Martinez et al. (2014) demonstrated that viscosity reduction associated with high-speed flagellar rotation provides an alternative explanation. The present model does not support a change to mean swimming speed purely through fluid anisotropy; because we analysed only 2D propulsion with constant viscosity-like parameters, we are unable to comment on the effect of shear-thinning on rotation.

The passive region of parameter space with $\mu_{1}=0$ represents the anisotropic characteristics of the aligned passive microstructure of cervical mucus. Key aspects that may be explored in future work include shear-dependent viscosity and dispersion of fibre alignment. The active regime $\mu_{1} \neq 0$ may be considered as a model of motility through an active aligned medium, which may capture some of the essential physics of sperm migration through ciliated epithelium in the female reproductive tract. Our predictions could be tested experimentally by constructing an actuated membrane and studying the dynamics of an overlying suspension of swimming bacteria or microrods.

This study has opened up a number of exciting future research directions. These include (but are not limited to) investigating the effects of viscoelasticity (cf. Kruse et al., 2005), fibre dispersion (cf. Woodhouse \& Goldstein, 2012) and the presence of walls (cf. Katz, 1974). Similarly, coupling the flagellar elastic behaviour to the viscous fluid mechanics to determine the effect on the beat pattern (Riley \& Lauga, 2014), and a full 3D computational treatment of the problem would be of interest. The model may also be developed to apply to peristaltic pumping by taking into account a cylindrical tube geometry. Taylor's swimming sheet has inspired decades of research into biological propulsion; the study presented here shows that Taylor's model continues to enable insight into novel areas of active fluid mechanics.

\section{Acknowledgements}

G.C. is supported by a Biotechnology and Biological Sciences Research Council (BBSRC) Industrial CASE Studentship (BB/L015587/1). The authors acknowledge C. Spalding and A. Tisbury for their contributions to an initial student project leading to this problem, and C. Holloway, Dr M. Gallagher and Dr M. Hicks for valuable discussions.

\section{Appendix A. Components of the stress tensor}

Assuming that the velocity takes the form $\boldsymbol{u}=(u(x, y, t), v(x, y, t))$, the components of the stress tensor are calculated as

$$
\begin{aligned}
\sigma_{11}= & -p+\mu_{1}(\cos \phi-\theta \sin \phi)^{2}+\left(2+\mu_{2}(\cos \phi-\theta \sin \phi)^{4}\right. \\
& \left.+4 \mu_{3}(\cos \phi-\theta \sin \phi)^{2}\right) \frac{\partial u}{\partial x}+\left(\mu_{2}(\cos \phi-\theta \sin \phi)^{3}(\sin \phi+\theta \cos \phi)\right.
\end{aligned}
$$




$$
\begin{aligned}
& \left.+2 \mu_{3}(\cos \phi-\theta \sin \phi)(\sin \phi+\theta \cos \phi)\right)\left(\frac{\partial u}{\partial y}+\frac{\partial v}{\partial y}\right) \\
& +\mu_{2}(\cos \phi-\theta \sin \phi)^{2}(\sin \phi+\theta \cos \phi)^{2} \frac{\partial v}{\partial y}, \\
\sigma_{12}= & \sigma_{21} \\
= & \mu_{1}(\cos \phi-\theta \sin \phi)(\sin \phi+\theta \cos \phi) \\
& +\left(1+\mu_{2}(\cos \phi-\theta \sin \phi)^{2}(\sin \phi+\theta \cos \phi)^{2}+\mu_{3}\left(1-\theta^{2}\right)\right)\left(\frac{\partial u}{\partial y}+\frac{\partial v}{\partial x}\right) \\
& +\mu_{2}(\cos \phi-\theta \sin \phi)^{3}(\sin \phi+\theta \cos \phi) \frac{\partial u}{\partial x} \\
& +\mu_{2}(\cos \phi-\theta \sin \phi)(\sin \phi+\theta \cos \phi)^{3} \frac{\partial v}{\partial y}, \\
\sigma_{22} & -p+\mu_{1}(\sin \phi+\theta \cos \phi)^{2}+\left(2+\mu_{2}(\sin \phi+\theta \cos \phi)^{4}\right. \\
& \left.+4 \mu_{3}(\sin \phi+\theta \cos \phi)^{2}\right) \frac{\partial v}{\partial y}+\left(\mu_{2}(\cos \phi-\theta \sin \phi)(\sin \phi+\theta \cos \phi)^{3}\right. \\
& \left.\times 2 \mu_{3}(\cos \phi-\theta \sin \phi)(\sin \phi+\theta \cos \phi)\right)\left(\frac{\partial u}{\partial y}+\frac{\partial v}{\partial x}\right) \\
& +\mu_{2}(\cos \phi-\theta \sin \phi)^{2}(\sin \phi+\theta \cos \phi)^{2} \frac{\partial u}{\partial x} .
\end{aligned}
$$

\section{Appendix B}

The components of the matrix $\boldsymbol{L}$ are calculated as

$$
\begin{aligned}
L_{11}= & \left(1+\frac{\mu_{2}}{4} \sin ^{2} 2 \phi+\mu_{3}\right)\left(\lambda^{4}-2 \lambda^{2}+1\right)-\mu_{2} \lambda^{2} \cos 4 \phi \\
& +\mu_{1}\left[2 \sin 2 \phi\left(\lambda \cos ^{2} \phi-\lambda^{3} \sin ^{2} \phi\right)+\left(\lambda+\lambda^{3}\right) \cos 2 \phi \sin 2 \phi\right], \\
L_{12}= & -\mu_{1}\left[2 \lambda^{2} \sin ^{2} 2 \phi+\cos 2 \phi\left(\left(\lambda^{2}+\lambda^{4}\right) \sin ^{2} \phi-\left(1+\lambda^{2}\right) \cos ^{2} \phi\right)\right] \\
& +\frac{\mu_{2}}{2}\left(\lambda^{3}+\lambda\right) \sin 4 \phi,
\end{aligned}
$$

where $L_{22}=L_{11}$ and $L_{21}=-L_{12}$.

\section{Appendix C}

The balance of (3.3) at order $\varepsilon^{2}$ is given by

$$
\begin{aligned}
& \left(1+\frac{\mu_{2}}{4} \sin ^{2} 2 \phi+\mu_{3}\right) \nabla^{4} \psi_{1}-\mu_{1}\left(2 \sin 2 \phi \frac{\partial^{2} \theta_{1}}{\partial x \partial y}+\cos 2 \phi\left(\frac{\partial^{2} \theta_{1}}{\partial x^{2}}-\frac{\partial^{2} \theta_{1}}{\partial y^{2}}\right)\right) \\
& +\mu_{2}\left(\cos 4 \phi \frac{\partial^{4} \psi_{1}}{\partial x^{2} \partial y^{2}}+\frac{\sin 4 \phi}{2}\left(\frac{\partial^{4} \psi_{1}}{\partial x \partial y^{3}}-\frac{\partial^{4} \psi_{1}}{\partial x^{3} \partial y}\right)\right) \\
& -\mu_{1}\left[2 \sin 2 \phi\left(\theta_{0}\left(\frac{\partial^{2} \theta_{0}}{\partial y^{2}}-\frac{\partial^{2} \theta_{0}}{\partial x^{2}}\right)+\left(\frac{\partial \theta_{0}}{\partial y}\right)^{2}-\left(\frac{\partial \theta_{0}}{\partial x}\right)^{2}\right)\right. \\
& \left.+\cos 2 \phi\left(4\left(\frac{\partial \theta_{0}}{\partial x} \frac{\partial \theta_{0}}{\partial y}+\theta_{0} \frac{\partial^{2} \theta_{0}}{\partial x \partial y}\right)\right)\right]-\mu_{2}\left[\operatorname { s i n } 4 \phi \left(2 \frac{\partial^{2} \theta_{0}}{\partial x \partial y} \frac{\partial^{2} \psi_{0}}{\partial x \partial y}\right.\right.
\end{aligned}
$$




$$
\begin{aligned}
& -\frac{\theta_{0}}{2}\left(\frac{\partial^{4} \psi_{0}}{\partial x^{4}}-3 \frac{\partial^{4} \psi_{0}}{\partial x^{2} \partial y^{2}}+\frac{\partial^{4} \psi_{0}}{\partial y^{4}}\right)+\frac{1}{2}\left(\frac{\partial^{2} \theta_{0}}{\partial x^{2}}-\frac{\partial^{2} \theta_{0}}{\partial y^{2}}\right)\left(\frac{\partial^{2} \psi_{0}}{\partial y^{2}}-\frac{\partial^{2} \psi_{0}}{\partial x^{2}}\right) \\
& \left.-\frac{\partial \theta_{0}}{\partial y}\left(\frac{\partial^{3} \psi_{0}}{\partial y^{3}}-3 \frac{\partial^{3} \psi_{0}}{\partial x^{2} \partial y}\right)+\frac{\partial \theta_{0}}{\partial x}\left(3 \frac{\partial^{3} \psi_{0}}{\partial x \partial y^{2}}-\frac{\partial^{3} \psi_{0}}{\partial x^{3}}\right)+\theta_{0} \frac{\partial^{4} \psi_{0}}{\partial x^{2} \partial y^{2}}\right) \\
& +\cos 4 \phi\left(2 \theta_{0}\left(\frac{\partial^{4} \psi_{0}}{\partial x^{3} \partial y}-\frac{\partial^{4} \psi_{0}}{\partial x \partial y^{3}}\right)+\left(\frac{\partial^{2} \theta_{0}}{\partial x^{2}}-\frac{\partial^{2} \theta_{0}}{\partial y^{2}}\right) \frac{\partial^{2} \psi_{0}}{\partial x \partial y}\right. \\
& -\frac{\partial \theta_{0}}{\partial x}\left(\frac{\partial^{3} \psi_{0}}{\partial y^{3}}-3 \frac{\partial^{3} \psi_{0}}{\partial x^{2} \partial y}\right)-\frac{\partial \theta_{0}}{\partial y}\left(3 \frac{\partial^{3} \psi_{0}}{\partial x \partial y^{2}}-\frac{\partial^{3} \psi_{0}}{\partial x^{3}}\right) \\
& \left.\left.-\frac{\partial^{2} \theta_{0}}{\partial x \partial y}\left(\frac{\partial^{2} \psi_{0}}{\partial y^{2}}-\frac{\partial^{2} \psi_{0}}{\partial x^{2}}\right)\right)\right]=0 .
\end{aligned}
$$

\section{Supplementary movies}

Supplementary movies are available at https://doi.org/10.1017/jfm.2016.821.

\section{REFERENCES}

Berg, H. C. \& TURner, L. 1979 Movement of microorganisms in viscous environments. Nature 278, 349-351.

Ceric, F., Silva, D. \& Vigil, P. 2005 Ultrastructure of the human periovulatory cervical mucus. J. Electron. Microsc. 54 (5), 479-484.

Chaudhury, T. K. 1979 On swimming in a visco-elastic liquid. J. Fluid Mech. 95 (01), 189-197.

Chrétien, F. C. 1982 Sperm cell-cervical mucus interaction. In Atlas of Human Reproduction (ed. E. S. E. Hafez \& P. Kenemans), pp. 219-222. Springer.

Chrispell, J. C., FAuCi, L. J. \& Shelley, M. 2013 An actuated elastic sheet interacting with passive and active structures in a viscoelastic fluid. Phys. Fluids 25 (1), 013103.

Drummond, J. E. 1966 Propulsion by oscillating sheets and tubes in a viscous fluid. J. Fluid Mech. 25 (04), 787-793.

Dyson, R. J., Green, J. E. F., Whiteley, J. P. \& Byrne, H. M. 2015 An investigation of the influence of extracellular matrix anisotropy and cell-matrix interactions on tissue architecture. J. Math. Biol. 72, 1775-1809.

Dyson, R. J. \& JENSEN, O. E. 2010 A fibre-reinforced fluid model of anisotropic plant cell growth. J. Fluid Mech. 655, 472-503.

Ericksen, J. L. 1960 Transversely isotropic fluids. Colloid Polym. Sci. 173 (2), 117-122.

FAUCI, L. J. \& Dillon, R. 2006 Biofluidmechanics of reproduction. Annu. Rev. Fluid Mech. 38, 371-394.

Fu, H. C., Powers, T. R. \& Wolgemuth, C. W. 2007 Theory of swimming filaments in viscoelastic media. Phys. Rev. Lett. 99 (25), 258101.

Fu, H. C., Wolgemuth, C. W. \& Powers, T. R. 2009 Swimming speeds of filaments in nonlinearly viscoelastic fluids. Phys. Fluids 21 (3), 033102.

Green, J. E. F. \& Friedman, A. 2008 The extensional flow of a thin sheet of incompressible, transversely isotropic fluid. Eur. J. Appl. Maths 19 (03), 225-257.

Holloway, C. R., Cupples, G., Smith, D. J., Green, J. E. F., Clarke, R. J. \& Dyson, R. J. 2016 Fundamental connections between models of active suspensions and transversely-isotropic fluids. arXiv:1607.00316.

Holloway, C. R., Dyson, R. J. \& SMith, D. J. 2015 Linear Taylor-Couette stability of a transversely isotropic fluid. Proc. R. Soc. Lond. A 471 (2178), 20150141.

Jeffery, G. B. 1922 The motion of ellipsoidal particles immersed in a viscous fluid. Proc. R. Soc. Lond. A 102 (715), 161-179. 
KATZ, D. F. 1974 On the propulsion of micro-organisms near solid boundaries. J. Fluid Mech. 64 (01), 33-49.

Krieger, M. S., Dias, M. A. \& Powers, T. R. $2015 a$ Minimal model for transient swimming in a liquid crystal. Eur. Phys. J. E 38 (8), 1-9.

Krieger, M. S., Spagnolie, S. E. \& Powers, T. $2015 b$ Microscale locomotion in a nematic liquid crystal. Soft Matt. 11 (47), 9115-9125.

Krieger, M. S., Spagnolie, S. E. \& Powers, T. R. 2014 Locomotion and transport in a hexatic liquid crystal. Phys. Rev. E 90 (5), 052503.

Kruse, K., Joanny, J.-F., JÜlicher, F., Prost, J. \& Sekimoto, K. 2005 Generic theory of active polar gels: a paradigm for cytoskeletal dynamics. Eur. Phys. J. E 16 (1), 5-16.

Lauga, E. 2007 Propulsion in a viscoelastic fluid. Phys. Fluids 19 (8), 083104.

Lauga, E. \& Powers, T. R. 2009 The hydrodynamics of swimming microorganisms. Rep. Prog. Phys. 72 (9), 096601.

Leshansky, A. M. 2009 Enhanced low-Reynolds-number propulsion in heterogeneous viscous environments. Phys. Rev. E 80 (5), 051911.

López, H. M., Gachelin, J., Douarche, C., Auradou, H. \& Clément, E. 2015 Turning bacteria suspensions into superfluids. Phys. Rev. Lett. 115 (2), 028301.

MagariYama, Y. \& KUdo, S. 2002 A mathematical explanation of an increase in bacterial swimming speed with viscosity in linear-polymer solutions. Biophys. J. 83 (2), 733-739.

Martinez, V. A., Schwarz-Linek, J., Reufer, M., Wilson, L. G., Morozov, A. N. \& Poon, W. C. K. 2014 Flagellated bacterial motility in polymer solutions. Proc. Natl Acad. Sci. USA 111 (50), 17771-17776.

PaK, O. S. \& Lauga, E. 2010 The transient swimming of a waving sheet. Proc. R. Soc. Lond. A 466 (2113), 107-126.

Riley, E. E. \& LAUGA, E. 2014 Enhanced active swimming in viscoelastic fluids. Europhys. Lett. 108 (3), 34003.

Riley, E. E. \& LAUGA, E. 2015 Small-amplitude swimmers can self-propel faster in viscoelastic fluids. J. Theor. Biol. 382, 345-355.

SAintillan, D. 2010 The dilute rheology of swimming suspensions: a simple kinetic model. Exp. Mech. 50 (9), 1275-1281.

SCHNeIDeR, W. R. \& Doetsch, R. N. 1974 Effect of viscosity on bacterial motility. J. Bacteriol. 117 (2), 696-701.

TAYlor, G. 1951 Analysis of the swimming of microscopic organisms. Proc. R. Soc. Lond. A 209 (1099), 447-461.

TAYLOR, G. I. 1952 The action of waving cylindrical tails in propelling microscopic organisms. Proc. R. Soc. Lond. A 211 (1105), 225-239.

Teran, J., Fauci, L. \& Shelley, M. 2010 Viscoelastic fluid response can increase the speed and efficiency of a free swimmer. Phys. Rev. Lett. 104 (3), 038101.

VÉlez-Cordero, J. R. \& LAUGA, E. 2013 Waving transport and propulsion in a generalized Newtonian fluid. J. Non-Newtonian Fluid Mech. 199, 37-50.

Woodhouse, F. G. \& Goldstein, R. E. 2012 Spontaneous circulation of confined active suspensions. Phys. Rev. Lett. 109 (16), 168105.

Wróbel, J. K., Lynch, S., BArrett, A., FAuCI, L. \& Cortez, R. 2016 Enhanced flagellar swimming through a compliant viscoelastic network in Stokes flow. J. Fluid Mech. 792, $775-797$. 